\title{
Machine Learning (ML) based Thermal Management for Cool- ing of Electronics Chips by Utilizing Thermal Energy Storage (TES) in Packaging that Leverage Phase Change Materials (PCM)
}

\author{
Aditya Jayakumar Chuttar ${ }^{1}$, Debjyoti Banerjee ${ }^{1-5, *}$ \\ 1 J. Mike Walker '66 Department of Mechanical Engineering, Texas A\&M University; \\ MS 3123 TAMU, College Station, TX 77843-3123; adityajchuttar@tamu.edu \\ 2 J. Mike Walker '66 Department of Mechanical Engineering, Texas A\&M University; \\ 3 Harold Vance Department of Petroleum Engineering, College of Engineering; \\ 4 Inter-Collegiate School of Engineering-Medicine (ENMED); \\ 5 Department of Medical Education, College of Medicine; \\ MS 3123 TAMU, College Station, TX 77843-3123; dbanerjee@tamu.edu \\ * Correspondence: dbanerjee@tamu.edu;
}

\begin{abstract}
Miniaturization of electronics devices is often limited by the concomitant high heat fluxes (cooling load) and maldistribution of temperature profiles (hot spots). Thermal energy storage (TES) platforms providing supplemental cooling can be a cost-effective solution, that often leverages phase change materials (PCM). Although salt hydrates provide higher storage capacities and power ratings (as compared to that of the organic PCMs), they suffer from reliability issues (e.g., supercooling). 'Cold Finger Technique (CFT)' can obviate supercooling by maintaining a small mass fraction of the PCM in solid state for enabling spontaneous nucleation. Optimization of CFT necessitates real-time forecasting of the transient values of the melt-fraction. In this study artificial neural network (ANN) is explored for real-time prediction of the time remaining to reach a target value of melt-fraction based on the prior history of the spatial distribution of the surface temperature transients. Two different approaches were explored for training the ANN model, using: (1) transient PCM-temperature data; or (2) transient surface-temperature data. When deployed in a heat sink that leverages PCM based passive thermal management systems for cooling of electronic chips and packages, this maverick approach (using the second method) affords cheaper costs, better sustainability, higher reliability and resilience.
\end{abstract}

Keywords: thermal management, electronics cooling, thermal energy storage, TES, duty cycle, phase change materials, PCM, cold finger technique, CFT.

\section{Introduction}

Rapid miniaturization of electronics device-elements has accelerated their performance and efficacy in commercial products. This has led to higher rates of heat dissipation which is a limiting factor in maximizing their performance. Semiconductor reliability, product life-time and performance diminish at elevated operating temperatures. Maldistribution of temperature ("hot spots") can lead to early and catastrophic failures also. Higher junction temperatures due to the hot spots result in a shortened lifespan of a semiconductor device. In fact, the chances of failure increase exponentially, if a local area on a chip heats up beyond the specified temperature [1]. This result is based on the widely known Black's equation [2]. Moreover, integration - a direct consequence of miniaturisation -has resulted in non-uniform heat flux generation. The progression of the state of the art of semiconductor technologies has largely followed the famous Moore's law [3]- 
which simply states that the number of transistors on a microchip will double every two years or so. However, the doubling rate has started to falter in recent times, primarily due to the failure of the packaging industries in meeting the heat dissipation requirements [4]. Removal of high heat flux at appropriate temperatures is a constant challenge faced by the electronics industry. Researchers have considered varied approaches for electronics cooling over the years [5,6]. Hence, to extract maximum efficacy from these innovations that are accruing from progressive miniaturization of electronics devices and packages into increasingly smaller form factors - better thermal management strategies and paradigms are needed to address the higher cooling loads (and for mitigating the localized transient hot spots).

\subsection{PCMs for Electronics Cooling}

Traditional cooling techniques typically consist of external air-cooled heat sinks, and are inadequate for satisfying the cooling loads from modern electronics. Emerging technologies include (i) Heat pipes; (ii) Heat pumps; (iii) Microchannels; (iv) Spray cooling; (v) Phase change material (PCM) based cooling; (vi) Free cooling; and (vii) Thermoelectric cooling [7]. A number of studies on these techniques have enabled the rank ordering of their efficacies [8-10]. Among these, passive cooling (e.g., leveraging PCMs) have garnered significant attention in recent times due to their cost-effectiveness and simplicity. Desirable properties of these PCM (e.g., high latent heat, narrow operating temperature envelope, high specific heat, and small volume expansion on melting) translate into compact form factors of the electronics chips and packages. A numerical study exploring novel design of a heat sink utilizing hollow aluminium pin fins filled with PCM showed that a small mass of PCM could effectively stabilize the microprocessor temperatures [11]. An experimental study on cooling of a personal digital assistant (PDA) using n-eicosane as a PCM for thermal energy storage (TES) showed that this architecture stabilized the junction temperatures and the effectiveness of such a heat storage unit depends on the amount of PCM used [12]. PCMs can be used for thermal control of electronics devices that have intermittent duty cycles, such as for mobile phones, digital video cameras, wearable computers and video-gaming chips. The thermal resistance of the device and the power rating of the PCM mass (for charging and discharging cycles) are the critical design parameters in this type of a passive system [13]. A PCM based heat sink (where the PCM is deposited in the cavities of the sink) placed on a plastic quad flat package mounted on a printed circuit board was shown to have an improved cooling performance as compared to that of the case without PCM, especially at high power levels (q), i.e., q exceeding 2 W [14]. Another study reported that for a fixed volume of PCM in the heat sink: the operation time (time required for the heat sink to achieve a target temperature) was enhanced significantly, where the degree of enhancement was elevated at progressively higher power levels [15]. It has been established that combining a PCM based heat sink with forced air convection provides a superior strategy in reducing (or damping) the peak temperatures than that of a traditional air-cooled heat sink without PCM. Moreover, the higher TES capacity of the PCM (due to higher latent heat values) increases the time needed to reach the peak temperature. For effective thermal management of electronics, it is desirable that PCMs should possess the following characteristics and properties - (i) appropriate and narrow band of phase transition temperature, (ii) low volume expansion coefficients (and during phase change), (iii) elevated thermo-physical properties (e.g., thermal conductivity, latent heat, and specific heat capacity values), (iv) material compatibility (noncorrosive) and chemical stability (especially for repeated melting and freezing), (v) low/no supercooling during freezing, (vi) low cost and (vii) minimal environmental foot-print [16]. All of these criteria can be seldom met in one candidate PCM exclusively, and therefore, often a trade-off must be made for choosing the appropriate PCM for a given application

\subsection{Supercooling in PCMs}


PCMs can be categorised as: (1) organic, (2) inorganic, and (3) eutectics. Example of organic PCMs include paraffin, fats and fatty acid-based derivatives. Popular inorganic PCMs include - salt hydrates such as $\left[\mathrm{LiNO}_{3} \cdot 3\left(\mathrm{H}_{2} \mathrm{O}\right)\right]$ and metallics such as Gallium. Eutectics are often realized by utilizing a mixture of two or more PCMs that provide congruent melting and freezing points. Salt hydrates have a higher volumetric energy storage capacity $\left(40-125\left[\mathrm{kWh} / \mathrm{m}^{3}\right]\right)$ than paraffins $\left(40-60\left[\mathrm{kWh} / \mathrm{m}^{3}\right]\right)$ due to their high densities [17]. Higher thermal conductivity and thermal diffusivity values of inorganic PCMs also enable higher power ratings of the TES platforms. So, in systems where volume is a constraint, salt hydrates can be a better option. However, salt hydrates undergo incongruent melting and often require large supercooling to initiate nucleation. Supercooling is the phenomenon where solidification is achieved only when the PCM temperature is significantly lower than the thermodynamic phase transition temperature. At the fusion temperature, the rate of nucleation is very low. This limits the efficacy of a PCM heat sink - as the latent heat is not released.

Methods to address supercooling can be categorised into passive and dynamic techniques. Addition of nucleating agents is a widely studied passive technique to mitigate supercooling. Using Borax (e.g., at a mass fraction of $1.9 \%$ ) as a nucleating agent reduced the degree of supercooling for thickened Glauber's salt from $15^{\circ} \mathrm{C}$ to $3 \sim 4{ }^{\circ} \mathrm{C}$ [18]. Supercooling in sodium acetate trihydrate (SAT) was entirely obviated upon addition of aluminium nitride (AIN) nanoparticles at a mass fraction of $5 \%$ along with carboxyl methyl cellulose (CMC) as a thickening agent at a mass fraction $4 \%$ [19]. Supercooling in SAT can also be reduced by adding silver nanoparticles and was reported to vary as a function of the concentration of the nanoparticles [20]. Similarly, copper hydroxyl nitrate hydrate $(\mathrm{CHNH})$ catalyst enhances nucleation in lithium nitrate trihydrate (LNT). Models suggest that the similarity of the lattice structures of both the salt and the catalyst (or nucleators) determine their effectiveness on mitigating supercooling [21]. Zinc hydroxyl nitrate and zinc oxide nucleators were observed to reduce the supercooling in zinc nitrate trihydrate by $8.8^{\circ} \mathrm{C}$ to $8.2{ }^{\circ} \mathrm{C}$. respectively [22]. The effect of various nucleating agents on the degree of supercooling in salt hydrates has been extensively reviewed in multiple reports in the literature [23,24].

In contrast to the heterogeneous seeding techniques, the 'cold finger technique' (CFT) is an example of a homogenous nucleation technique where a small mass fraction of the salt hydrate crystals are maintained in a solid phase (un-melted) in contact with the liquid phase, thus aiding spontaneous nucleation when freezing is initiated. A localised spot (or tiny mass fraction) can be maintained in the solid phase by adding extra insulation or exerting localized pressure. Another way to implement the cold finger technique is to halt the melting process of the PCM just prior to complete melting. For instance, the heat source could be removed when the PCM reaches 85\% melt, leaving 15\% un-melted (in order to promote spontaneous nucleation during the freezing cycle). However, the enhanced reliability in CFT is achieved at the expense of reduced energy storage capacity.

Combining CFT with a forecasting technique (for predicting the time required to reach a target melt fraction) can successfully mitigate reliability issues by obviating supercooling while maximizing the energy storage capacity (and to some extent the power rating) of the TES platform utilizing inorganic PCMs. For a TES application requiring repeated thermocycling (i.e., repeated consecutive cycles of melting and freezing), CFT was demonstrated to be effective in reducing the degree of supercooling in LNT (from $\sim 10{ }^{\circ} \mathrm{C}$ ) to less than $1^{\circ} \mathrm{C}$ (e.g., in the range of $0.5-1^{\circ} \mathrm{C}$ ). The authors demonstrated that using the CFT technique the LNT samples survived thermocycling tests that exceeded 800 cycles of repeated incomplete melting and complete solidification [25].

Motivated by the goal of improving the effectiveness of inorganic PCMs incorporated into TES platforms that can be deployed for electronics chip cooling applications, in this study the efficacy of deep learning is explored for enhancing the energy storage capacity for CFT by forecasting the time required to reach a target melt-fraction (say, 85\% or 99\%). In this study, a graduated measuring cylinder was filled with PCM and an electrically powered heater was mounted at the bottom of the cylinder to melt the PCM. 
Thermocouples were mounted within the body of the PCM as well as on the surface of the measuring cylinder at specific locations. A digital data acquisition system was used to record the temperature transients from the thermocouples. The temperature data were fed to an ANN model with the goal of performing real-time predictions for the time required to reach a target melt-fraction (e.g., 85\%). Hence, artificial neural network (ANN) models were developed in this study for real-time prediction of the time remaining to reach a predefined (or target) melt-fraction at any instant during the experiments as the PCM melts. This study was performed to contrast the efficacy of two different approaches utilizing the same ANN model:

(1) using temperature data from sensors immersed within the body of the PCM at locations corresponding to meniscus levels of the liquid coinciding with specific values of melt-fraction of the PCM (i.e., transient PCM-temperature data for training of the ANN model); and

(2) using temperature data from sensors mounted on the surface of the cylindrical container at locations corresponding to meniscus levels of the liquid coinciding with specific values of melt-fraction of the PCM (i.e., transient surface-temperature data for training of the ANN model).

The efficacy of these two approaches is compared for different values of the electrical power input to the heater (that is mounted at the bottom of the cylindrical container). The error in the predictions from the ANN model (compared to the actual values recorded in the experiments) are explored for different sets of training data obtained from these experiments.

\section{Artificial Neural Network Principles}

Artificial neural networks are capable of fitting complex, non-linear mappings between inputs and outputs [26]. An ANN can be defined as a parallel distributed processing system capable of learning from experiences [27]. ANNs are built to construct relationships between parameters without detailed knowledge about the system. The most basic kind of an ANN is the fully connected multilayer perceptron (MLP) model. In such networks, the neuron is the fundamental processor of information. These neurons (also referred to as 'nodes') are arranged in progressive layers. In case of a fully connected MLP, a neuron receives and processes the input from the neurons in the previous layer into an output. A neuron can be mathematically characterised by: (i) bias (b); (ii) activation function $(f)$. Each synaptic connection is associated with a weight $(w)$. Inside every node the activation function acts on the weighted inputs from the previous layer. Let the subscript $n$ denote the serial number of the layer. The input vector to the layer can be represented by $a_{n}$. The relationship between the output (the input to $a_{n+1}$ ) is depicted in Equation (1) below:

$$
a_{n+1}=f\left(w^{T} a_{n}+b\right)
$$

Developing an ANN model involves training, validation, and testing stages. The input data and the output (predictions) can be divided into three sets, one for each stage. The weights and biases are randomly initialised. The output (i.e., the set of predicted value[s] from the ANN model) is obtained using these random parameters. A cost function then quantifies the degree of disagreement (or error) between the generated (predicted) output and the actual (true) output, thus going through the validation step. This obtained error is propagated backwards from the output to the input layer by adjusting the weights and biases. A gradient descent algorithm is applied to modify the biases and weights to optimize (minimize) the cost function. This feedforward and backpropagation process continue until the target error (or target gradient) is achieved or the number of passes (epochs) reaches a specified value. A typical example of the cost function is the "sum of squared errors" (SSE) on the validation dataset depicted in (2) where $p$ is the true value and $p^{\prime}$ is the value predicted by the ANN model. Also, $N$ denotes the size of the test 
set. The third stage involves testing the efficacy of the ANN on the test dataset which was not encountered by the ANN during the training or the validation stage.

$$
S S E=\sum_{i=1}^{N}\left(p-p^{\prime}\right)^{2}
$$

The efficacy of a neural network for improving the reliability of a TES platform has been previously studied by the authors of this paper [28]. For electronics cooling applications, PCM heat sinks are an emerging solution. During intermittent operation of electronic devices - a heat sink filled with PCM can limit the peak value of the local temperature transients by rapid melting (faster discharging cycle) and release the heat when the device is idle (slow period of freezing, i.e., slower charging cycle). During thermocycling of PCM, the power rating is typically higher during the charging cycle (i.e., during melting, since it is dominated by free-convection heat transfer) and the power rating is lower during the discharging cycle (i.e., during freezing, since it is dominated by conduction heat transfer and convection is almost negligible) [25]. Thus, this strategy helps with extending the time required to reach the peak temperature of the heat sink, especially under critical load conditions [29].

As mentioned before, in this study, the prediction errors from the ANN model that is trained using transient surface-temperature data are compared with the same ANN model that is trained using temperature transients recorded by thermocouples immersed within the volume of the PCM. Measurement of surface temperature transients are preferable for electronics packages (filled with PCMs) due to their simplicity, low cost, enhanced reliability, as well as ease of manufacturing and fabrication. Such a capability can facilitate lower failure rates while also providing ease of access for maintenance operations and repair jobs. Forecasting strategies based on transient surface-temperature data also enable retrofitting of existing electronics devices with capabilities for real-time predictions using ANN models (e.g., the time remaining for reaching a target melt fraction of PCMs that are filled inside the electronics package). These capabilities do not typically accrue from strategies involving measurement of temperature transients from sensors that are immersed within the volume of PCM, i.e., from temperature sensors mounted inside the electronics package (instead of the surface mounted temperature sensors). Hence, this study demonstrates the efficacy of ANN models for electronics chip cooling applications and can be used in conjunction with PCM filled heat sinks to improve their reliability for thermal management applications (e.g., in data centres).

\section{Experimental Apparatus and Procedure}

Thermocycling experiments were conducted in this study to obtain a dataset consisting of temperature transients and the corresponding values of melt-fraction. PureTemp 29TM is chosen as the candidate PCM in these experiments. The salient properties of Pure Temp29TM are listed in Table 1. The data from these experiments were used for training an ANN model. The goals of these experiments and numerical simulations were focused on deploying the ANN model for real-time prediction of the time required to reach a target value of melt-fraction (e.g., 85\%). The errors in the predictions (compared to experimental data) were obtained from this study with the goal of identifying the ANN model that yields the best performance.

Solid sample of PCM was heated in a graduated measuring cylinder (with total volume of $50 \mathrm{ml}$ ) and with a least count of $1 \mathrm{ml}$. The experiments were performed until the PCM was melted completely. Broadly, the apparatus consists of four components: (i) the graduated measuring cylinder; (ii) K-type thermocouples; (iii) heater coil; and (iv) Data acquisition system.

The thermocouples were mounted at specific locations - both within the volume of the cylinder as well as on the outside surface of the cylinder. These locations were determined strategically in order to measure the temperatures at particular heights (from the bottom) and along the axis of the cylinder. The vertical heights were chosen strategically 
to correspond to the level of the liquid meniscus of the melted PCM for a pre-determined value of melt fraction.

Table 1. Salient Properties of PureTemp29 [30]

\begin{tabular}{cc}
\hline Property & Value \\
\hline Enthalpy of Phase Change & $202[\mathrm{~J} / \mathrm{g}]$ \\
\hline Heat Capacity of Solid & $1.77\left[\mathrm{~J} /\left(\mathrm{g}{ }^{\circ} \mathrm{C}\right)\right]$ \\
\hline Heat Capacity of Liquid & $1.94\left[\mathrm{~J} /\left(\mathrm{g}{ }^{\circ} \mathrm{C}\right)\right]$ \\
\hline Density of Solid & $0.94[\mathrm{~g} / \mathrm{ml}]$ \\
\hline Density of Liquid & $0.85[\mathrm{~g} / \mathrm{ml}]$ \\
\hline Conductivity of liquid & $0.15\left[\mathrm{~W} /\left(\mathrm{m}{ }^{\circ} \mathrm{C}\right)\right]$ \\
\hline Conductivity of solid & $0.25\left[\mathrm{~W} /\left(\mathrm{m}^{\circ} \mathrm{C}\right)\right]$ \\
\hline
\end{tabular}

The heater assembly is composed of a Nichrome wire (coil) connected to a DC power supply. The coil is placed at the bottom of the cylinder for melting the PCM for a fixed value of input power. The ends of the coil are connected to a DC supply by means of insulted connecting wires. Such a configuration means that when the liquid meniscus is at $0 \mathrm{ml}$ marking (i.e., there is no melting), the PCM is at a $\%$ melt-fraction. Whereas, when the liquid meniscus is at the $50 \mathrm{ml}$ marking, the PCM is at $100 \%$ melt-fraction. The thermocouples are placed at particular locations (within the volume of the PCM) and along the axis of the cylinder corresponding to $30 \%, 60 \%, 85 \%, 90 \%, 95 \%$, and $99 \%$ melt-fraction. On the outer surface of the measuring cylinder, the surface-mounted thermocouples are located at heights corresponding to $30 \%, 60 \%, 90 \%$ and $99 \%$ melt-fraction. In addition to these sets, another thermocouple is located at a distance of $1 \mathrm{~cm}$ above the $50 \mathrm{ml}$ mark on the outside surface of the measuring cylinder, i.e., at a distance of $1 \mathrm{~cm}$ above the height of the meniscus corresponding to $100 \%$ melt-fraction. Thermal paste (Omegabond ${ }^{\mathrm{TM}}$ ) is used as a glue to mount the thermocouples on the outside surface of the measuring cylinder. The thermocouples are calibrated in the temperature range of $20{ }^{\circ} \mathrm{C}$ to $40{ }^{\circ} \mathrm{C}$. The calibration step involves the measurement of the steady state temperature of each thermocouple when placed in a water bath and the temperature measurements were recorded by an automated digital data acquisition system for a period of 1 minute. The calibration was performed for fixed temperature values (of the water bath) corresponding to $20{ }^{\circ} \mathrm{C}$, $25{ }^{\circ} \mathrm{C}, 30{ }^{\circ} \mathrm{C}, 35^{\circ} \mathrm{C}$, and $40{ }^{\circ} \mathrm{C}$. The calibration was performed using a NIST calibrated mercury thermometer with a least count of $0.1^{\circ} \mathrm{C}$. The resultant calibration error (instrument/ bias error and statistical error) is estimated to be less than $\pm 0.1^{\circ} \mathrm{C}$ with a $68 \%$ statistical confidence (i.e., within one standard deviation of the average value).

\subsection{Volume Calibration Experiments}

The location of the thermocouples along the axis of the cylinders and its outer surface is based on the particular location of the liquid meniscus of the PCM (as it melts progressively) corresponding to chosen and specific values of melt-fraction of the PCM. A series of volume-calibration experiments were performed in the measuring cylinder (before performing the PCM experiments) in order to determine the height corresponding to a particular value of the volume of the liquid phase. This was performed by filling the measuring cylinder with a fixed quantity of water and noting the level of the liquid meniscus in the measuring cylinder. With the heater assembly and the thermocouple positioning apparatus inside the cylinder, water is poured in $5 \mathrm{ml}$ aliquots. The water level rise is recorded until the water reaches the $50 \mathrm{ml}$ mark. Due to the volume occupied by the apparatus inside, the volume level shown by the cylinder is higher than the actual volume of water in it. Using the densities of the solid and liquid PCM phases, the cylinder markings corresponding to the chosen melt fractions are determined. Interestingly, there exists a 
maximum value of the melt-fraction (less than 100\%) such that the thermocouple at that point remains inside the PCM mass throughout the experiment. This occurs due to PCM volume shrinkage. Upon solidification of the PCM, the thermocouples mounted at locations corresponding to higher values of melt fraction (which are initially submerged in the liquid PCM) are then exposed to air (instead of being submerged in the solidified mass of PCM) since the air-PCM interface recedes upon complete solidification of the PCM. In this experiment, thermocouples mounted at locations corresponding to melt-fraction exceeding 95\% - therefore - would not be immersed in the PCM during the entirety of the experiments. Hence, initially at the beginning of the melt-cycle these thermocouples would be exposed to air (and will record the temperature of the surrounding air). However, towards the final stages of the melt cycle (when the melt-fraction exceeds 95\%), these thermocouples will be submerged in the liquid PCM and would record the temperature of the liquid phase of the PCM.

\subsection{Data Acquisition (DAQ) System}

A digital data acquisition system (NI SCXI 1303 board with the NI SCXI 1000 chassis, from National Instruments Inc.) was used for recording the transient temperature data from the thermocouples. The data acquisition process was automated using Labview ${ }^{\mathrm{TM}}$ software (from National Instruments Inc.). The photograph of the experimental setup is shown in Fig. 1. Figure 2 schematically depicts the different components of the experimental apparatus. A plastic tube was inserted into the measuring cylinder (prior to filling it with PCM). The plastic tube was used for mounting the thermocouples and for the purpose of placing the beaded ends of the thermocouples at specific locations precisely along the vertical direction. The lower end of the plastic tube was sealed to prevent PCM from seeping in, and the thermocouple wires emerged out of the upper end of the plastic tube. The cylinder is filled with liquid PCM up to the $50 \mathrm{ml}$ mark and allowed to solidify under ambient conditions.

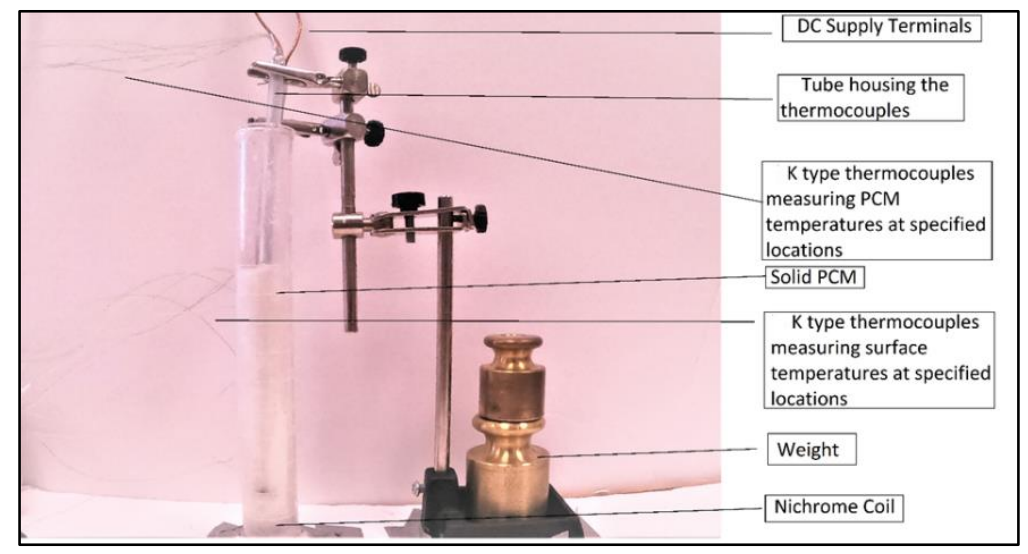

Figure 1. Photograph of the experimental apparatus used in this study. 


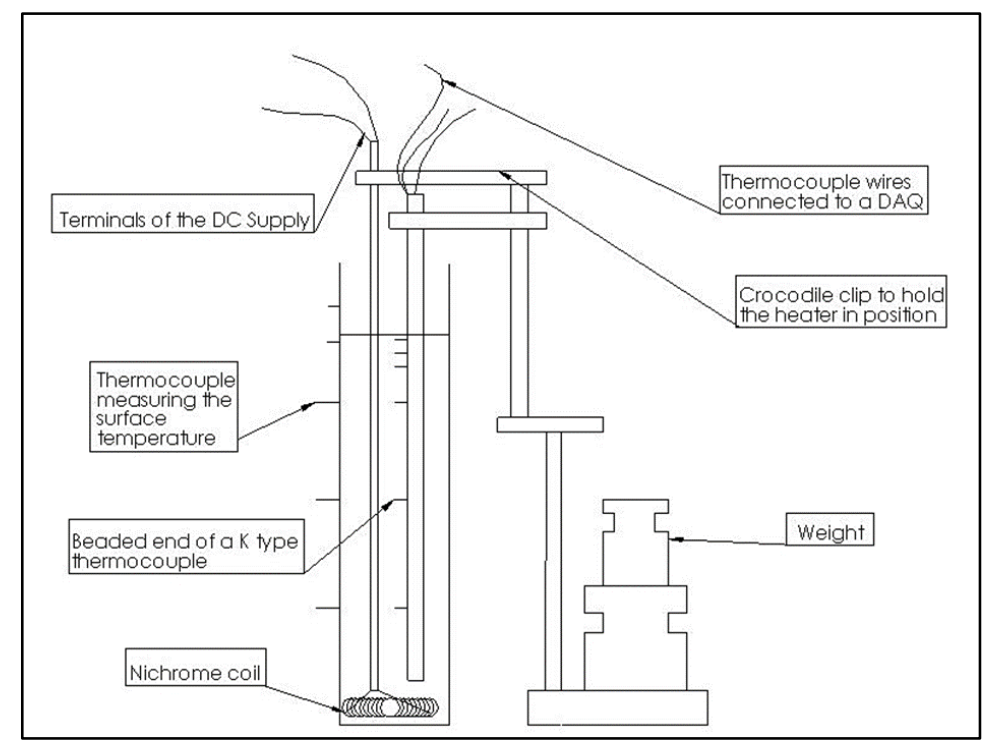

Figure 2. Schematic of the experimental apparatus used in this study.

Digital images of the whole volume of PCM within the measuring cylinder were captured every $60 \mathrm{~s}$ during the melting experiments in order to monitor the rise of the liquidsolid interface along the vertical direction. An infra-red (I.R.) camera was used to capture I.R. images intermittently at specific intervals of time. The I.R. images were used to monitor the temperature distribution and ascertain temperature uniformity within different parts of the experimental apparatus. Representative I.R. images are shown in Fig. 3 at different points in time. Using the apparatus, melting experiments were performed by setting the heater voltage at a constant value. The thermocouple and the visual data (digital images and IR images) were recorded until complete melting of the PCM was achieved.

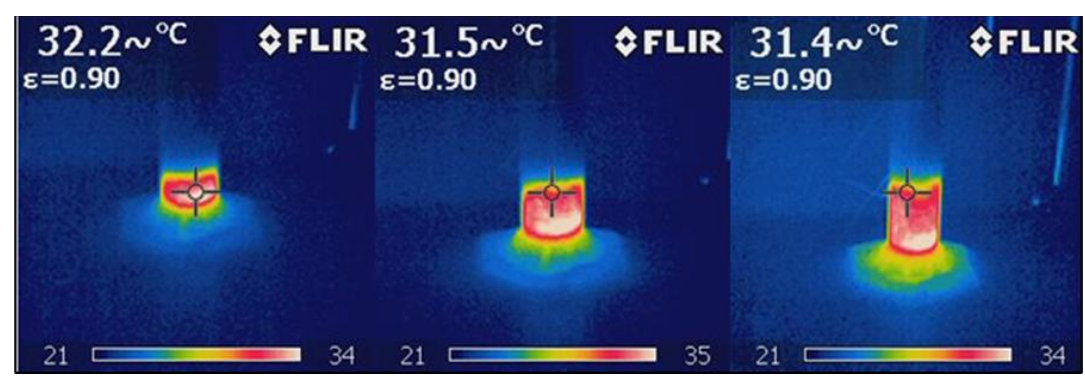

Figure 3. Images of the PCM undergoing melting in the cylinder for heater input power of $1.6 \mathrm{~W}$ (2.8 $\mathrm{V}$ input condition)

Experiments were performed for three sets of heater input voltages $-2.8 \mathrm{~V}(1.6 \mathrm{~W})$, $2.6 \mathrm{~V}(1.4 \mathrm{~W})$, and $2.3 \mathrm{~V}(1.08 \mathrm{~W})$. Each experiment was conducted twice to verify repeatability of the experimental data. The transient temperature data recorded by the thermocouples is plotted using a line graph. Fig. 4 shows the transient temperature profile recorded by the thermocouples that were mounted within the mass of PCM ("transient PCM-temperature data"). Fig. 5 shows the transient temperature profile recorded by thermocouples mounted on the surface of the measuring cylinder ("transient surface-temperature data"). In these plots, the abscissa denotes the elapsed time in seconds [s]. 


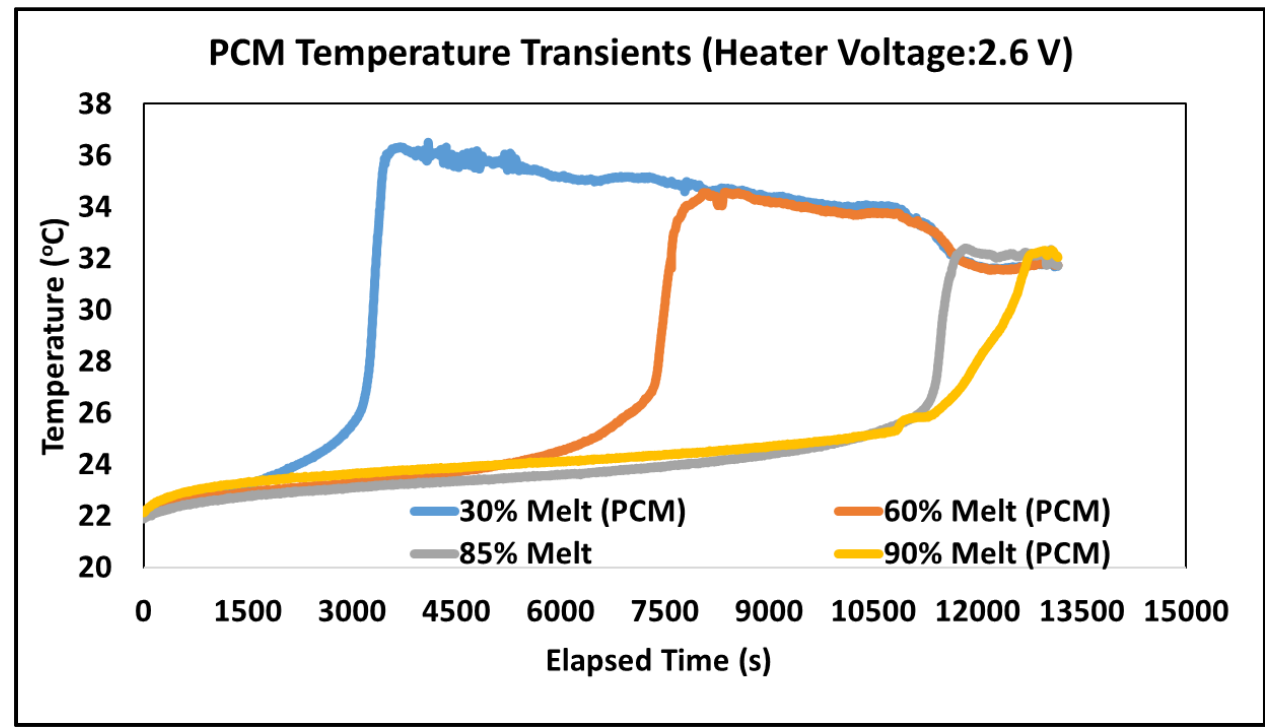

Figure 4. Plot of transient PCM-temperature data for heater input voltage of $2.6 \mathrm{~V}$ (1.4 W power input).

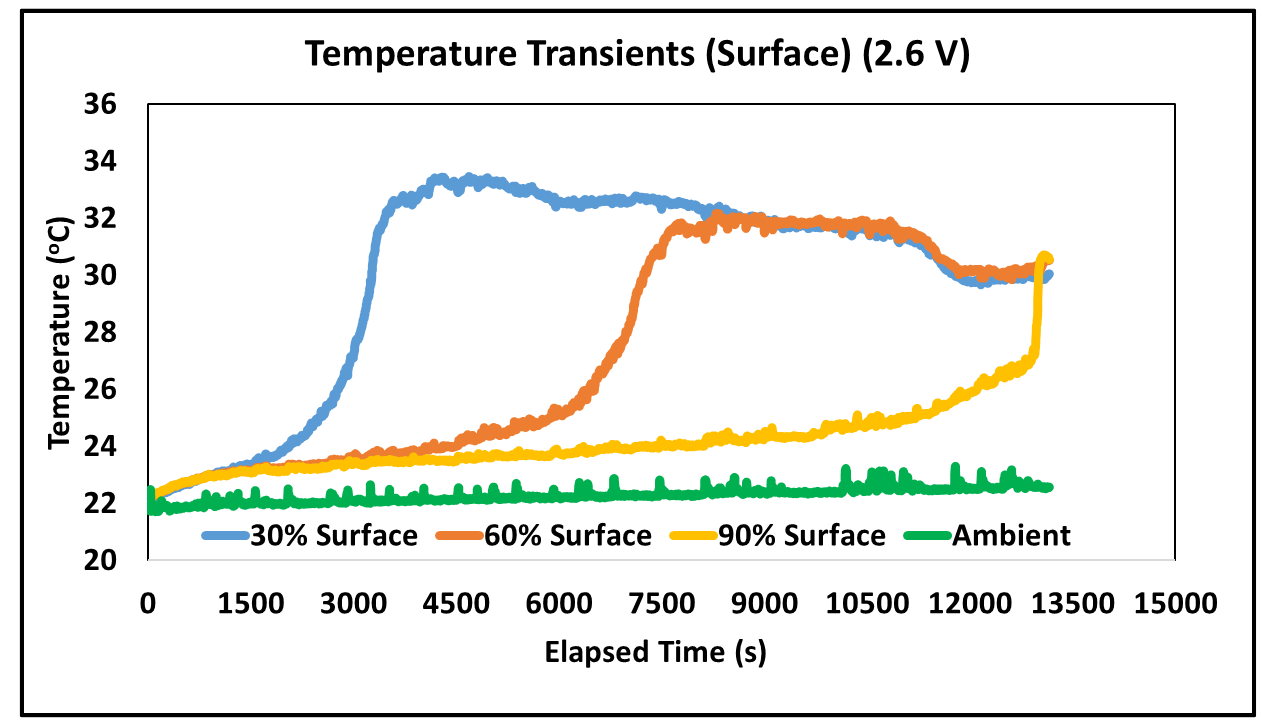

Figure 5. Plot of transient surface-temperature data for heater input voltage of $2.6 \mathrm{~V}$ (1.4 W power input).

The goal of this study was to predict in real-time (i.e., at any instant during the melting process) - the time remaining to attain $85 \%$ melt-fraction. In deep learning terms, the "time to reach $85 \%$ melt-fraction" is the label. This label is obtained by subtracting the time recorded corresponding to each temperature measurement (consisting of the following set: [ $\left.\left.T_{30}, T_{60}, T_{85}, T_{90}, T_{95}, T_{99}, T^{\prime}{ }_{30}, T^{\prime}{ }_{60}, T^{\prime}{ }_{90}, T^{\prime}{ }_{99}, T_{\text {ambient }}\right]\right)$ from the time when melt fraction of $85 \%$ is attained. The target value of $85 \%$ melt-fraction is achieved when the melt front hits the particular thermocouple inside the PCM mass (indicated by the sudden rise in temperature recorded by that particular thermocouple). This point is indicated by the temperature profile of that thermocouple (Fig. 4). The temperature curve begins to plateau after a sharp increase. The time stamp on this point serves our purpose for generating the labels.

A Multilayer Perceptron (MLP) Network is devised with three input nodes. Fig. 6 shows the topology of the neural network. The PCM temperatures, marked as: $T_{30}, T_{60}$ and $T_{90}$; constitute the three inputs. A nondimensional quantity, referred to as reduced time, is formulated. Nondimensionalizing the time parameter allows us to train the ANN using one experimental dataset and predict for another case. This reduced time, $\tau$, is obtained 
by taking the ratio of the elapsed time to the time when the target value $(85 \%$ melt-fraction) is achieved. It follows that the nondimensional form of the label is then the complementary value of $\tau$ (i.e., obtained by subtracting $\tau$ from unity and is denoted by $\tau^{\prime}$ ). Fig. 7 depicts the plot of temperatures as a function of nondimensional time $(\tau)$ and was obtained after the implementing the aforementioned steps (summarized in this paragraph). The three inputs to the ANN are (as mentioned before): $T_{30}, T_{60}$, and $T_{90}$. The output of the ANN is $\tau^{\prime}$. When exploring the performance of an ANN trained on surface temperatures, $T_{30}, T_{60}, T_{90}$ get replaced by surface temperatures $T^{\prime}{ }_{30}, T^{\prime}{ }_{60}$, and $T^{\prime}{ }_{90}$, respectively.

The MLP model (i.e., the artificial neural network/ ANN model) is trained using $\tau^{\prime}$ as the label and the three temperature inputs (recorded by the experiment for a particular power input). The training process is based on the well-known back-propagation algorithm which modifies the weights and biases of a node as it iterates through the dataset. The training process is stopped by using the 'early stopping' approach. In other words, when the MSE does not improve substantially upon further iterations, the training process is halted. The neural network in this study is composed of three hidden layers and each layer has 512 nodes. The Rectified Linear Unit (ReLU) activation function is used in the network nodes. The cost function for training purposes is the Mean Squared Error (MSE). ADAM optimizer is deployed as the gradient descent algorithm. The predictions are generated for all the six combinations of training/ prediction by utilizing the three datasets.

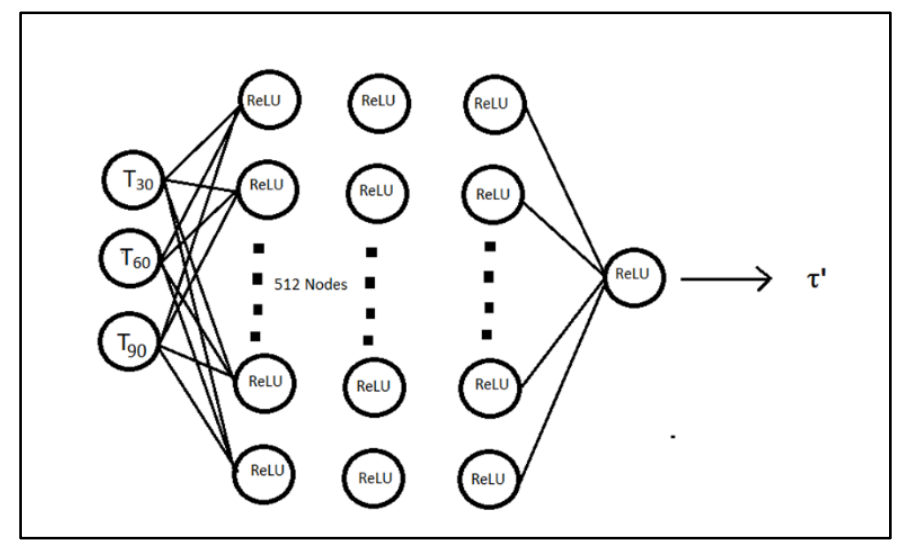

Figure 6. Topology of the MLP network used in this study.

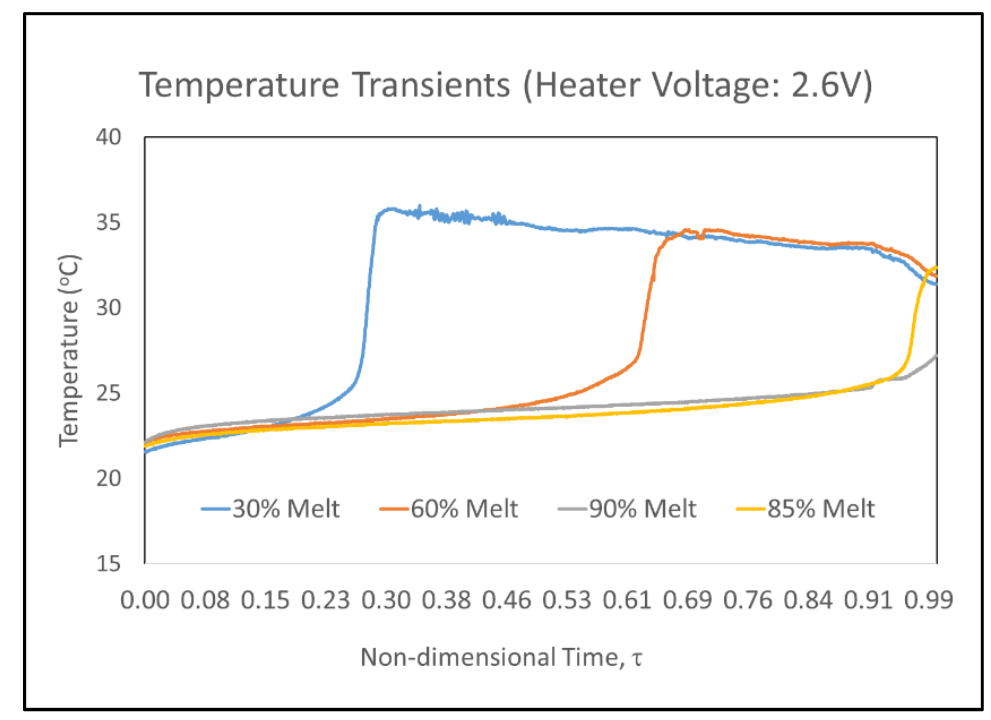

Figure 7. Temperature transients from the three thermocouples mounted within the mass of PCM in the experimental apparatus for a heater input of $2.6 \mathrm{~V}$, plotted as a function of the non-dimensional time $(\tau)$.

\section{Results and Discussion}


Table 2 lists the time taken to reach various melt-fractions for the three datasets. The experiments performed for $2.3 \mathrm{~V}$ input to the heater corresponds to a current of $0.47 \mathrm{~A}$ and a power input of $1.08 \mathrm{~W}$. When the heater input voltage is $2.6 \mathrm{~V}$, the heater draws a current $0.54 \mathrm{~A}$ and a power input of $1.4 \mathrm{~W}$. For $2.8 \mathrm{~V}$ input, the current and power inputs are $0.58 \mathrm{~A}$ and $1.6 \mathrm{~W}$, respectively. We observe that the time needed to attain $85 \%$ melt-fraction for the $2.3 \mathrm{~V}$ and $2.6 \mathrm{~V}$ experiments are quite similar. This is attributed to the ambient temperature effects. For the $2.3 \mathrm{~V}$ experiment, the average ambient temperature is observed to be $22.8^{\circ} \mathrm{C}$ (standard deviation $=0.2^{\circ} \mathrm{C}$ ) whereas for the $2.6 \mathrm{~V}$ experiment, the average recorded ambient temperature is $22.3^{\circ} \mathrm{C}$ (standard deviation $=0.2{ }^{\circ} \mathrm{C}$ ). The repeatability of the experiments can be established by plotting temperature transients recorded by a thermocouple, e.g., $T_{30}$ against non-dimensional time (where the total time spans from 0 to $100 \%$ melt). As an example, Figs. 8-10 shows the repeatability of the $2.8 \mathrm{~V}$ experiment. The initial temperature of the solid PCM at the beginning of the two instances of the $2.8 \mathrm{~V}$ experiment are different (due to different ambient conditions) causing the deviations observed in Figs. 8-10. The heat lost from the apparatus (due to free convection and radiation phenomena) is also influenced by the ambient temperature. Additional plots of the experimental data for the different power inputs are provided in the Appendix A.

Table 2. Time to reach specific values of melt-fraction for different values of power input to the heater.

\begin{tabular}{cccc}
\hline $\begin{array}{c}\text { Heater } \\
\text { Voltage } \\
{[\mathrm{V}]}\end{array}$ & $\begin{array}{c}\text { Time to reach } \\
\text { 85\% Melt-Fraction [s] }\end{array}$ & $\begin{array}{c}\text { Time to reach } \\
\text { 90\% Melt-Fraction [s] }\end{array}$ & $\begin{array}{c}\text { Time to reach 100\% } \\
\text { Melt-Fraction } \\
{[\mathrm{s}]}\end{array}$ \\
\hline $2.3 \mathrm{~V}$ & 12,017 & 13,670 & 15,658 \\
\hline $2.6 \mathrm{~V}$ & 11,817 & 12,729 & 13,157 \\
\hline $2.8 \mathrm{~V}$ & 8,768 & 9,888 & 10,518 \\
\hline
\end{tabular}

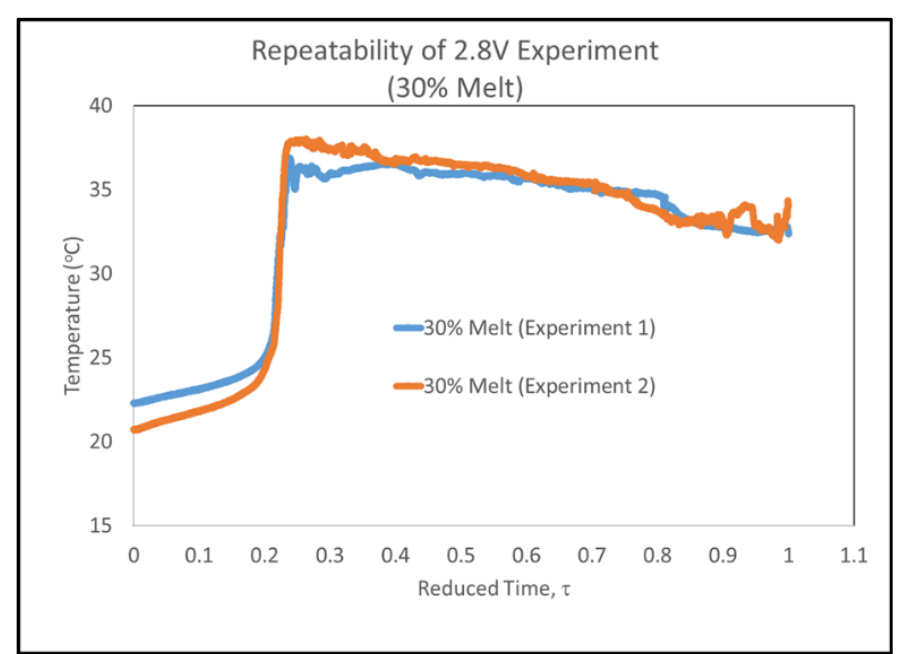

Figure 8. Comparison of temperature profiles in two different experiments for verifying the repeatability of the experiments: for the thermocouples mounted at the location corresponding to the height of the liquid meniscus for PCM melt-fraction of $30 \%$ and for heater input power of 1.6 $\mathrm{W}$ (2.8 V input). 


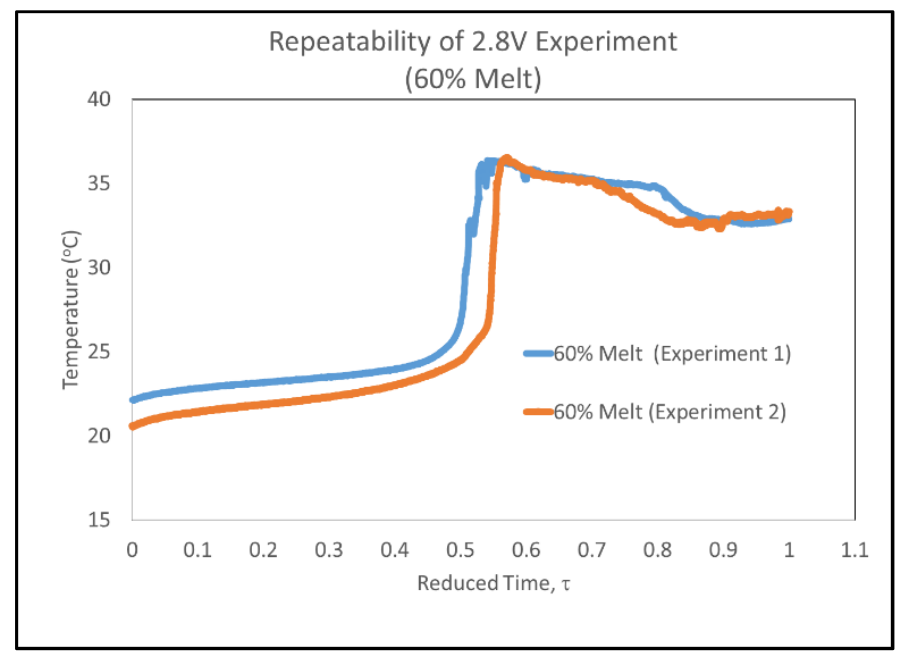

Figure 9. Comparison of temperature profiles in two different experiments for verifying the repeatability of the experiments: for the thermocouples mounted at the location corresponding to the height of the liquid meniscus for PCM melt-fraction of $60 \%$ and for heater input power of 1.6 W (2.8 V input).

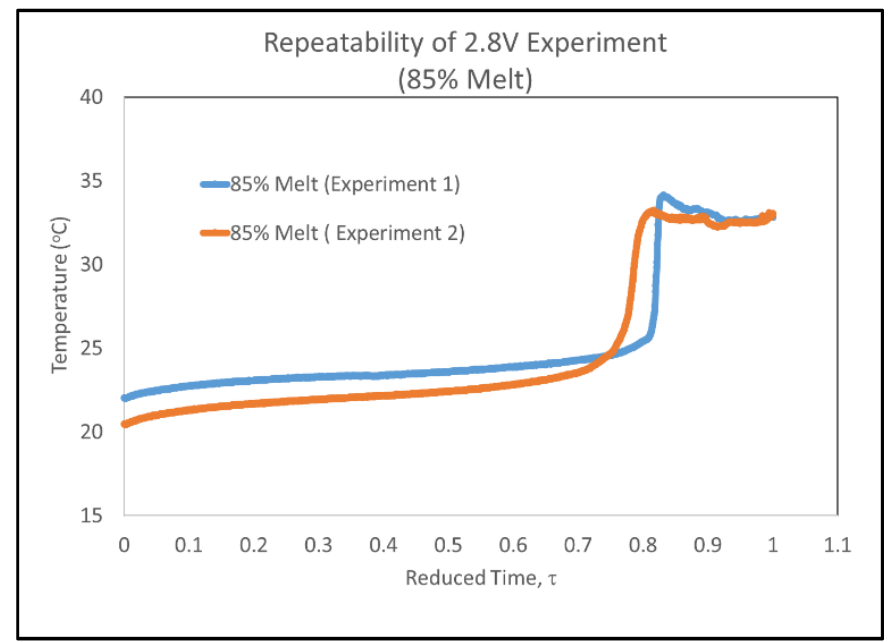

Figure 10. Comparison of temperature profiles in two different experiments for verifying the repeatability of the experiments: for the thermocouples mounted at the location corresponding to the height of the liquid meniscus for PCM melt-fraction of $85 \%$ and for heater input power of 1.6 $\mathrm{W}(2.8 \mathrm{~V}$ input).

\subsection{Performance of the ANN Trained on PCM Temperatures}

The three datasets $(2.3 \mathrm{~V}, 2.6 \mathrm{~V}$ and $2.8 \mathrm{~V}$ input) are used in all possible permutations of training and prediction sets. For instance, the temperature transients obtained from the thermocouples immersed in the PCM for $2.6 \mathrm{~V}$ input is used to train the neural network. This ANN is then used to predict the time remaining to reach $85 \%$ melt for the $2.8 \mathrm{~V}$ input condition (and the errors in the predicted values are calculated from the experimental data for the $2.8 \mathrm{~V}$ input condition). For a graphical representation, the predictions are depicted on a scatter plot for any combination of the training and prediction datasets. For instance, using the $2.6 \mathrm{~V}$ dataset for training and deploying the ANN (to obtain the predictions on the $2.8 \mathrm{~V}$ input condition) then yields the scatter plot in Fig. 11. The non-dimensional plot can be dimensionalized by multiplying the predicted $\tau^{\prime}$ values by the time-duration to attain $85 \%$ melt (from Table 2 ) and is depicted in Fig. 12 . The solid red line ( $y=x$ curve) serves as a reference for deviations of the predictions from the actual ("true" experimental) values. The dotted lines mark the point when the PCM reaches $30 \%$ and $60 \%$ meltfractions (which are registered by individual thermocouples mounted at each of those specific locations). On the non-dimensional scatter plot (e.g. Fig. 11), the green dotted line 
represents the point when the melt front reaches the respective thermocouple on the training set whereas the blue dotted line denotes the same on the prediction set. The prediction error is formulated as the difference between the predicted value and the actual value of time (in seconds). A low error is desired particularly at the final stages, i.e. when the PCM is about to reach a melt fraction of $85 \%$ so that the melting cycle can be halted in time in a real life application of this method. For a training/prediction combination of $2.6 \mathrm{~V} / 2.8 \mathrm{~V}$, the average absolute error in the final $1800 \mathrm{~s}$ ( 0.5 hours) before reaching $85 \%$ melt fraction is about 3 minutes $(167 \mathrm{~s})$ which is a minute fraction $(\sim 2 \%)$ of the total cycle time $(8768 \mathrm{~s})$.

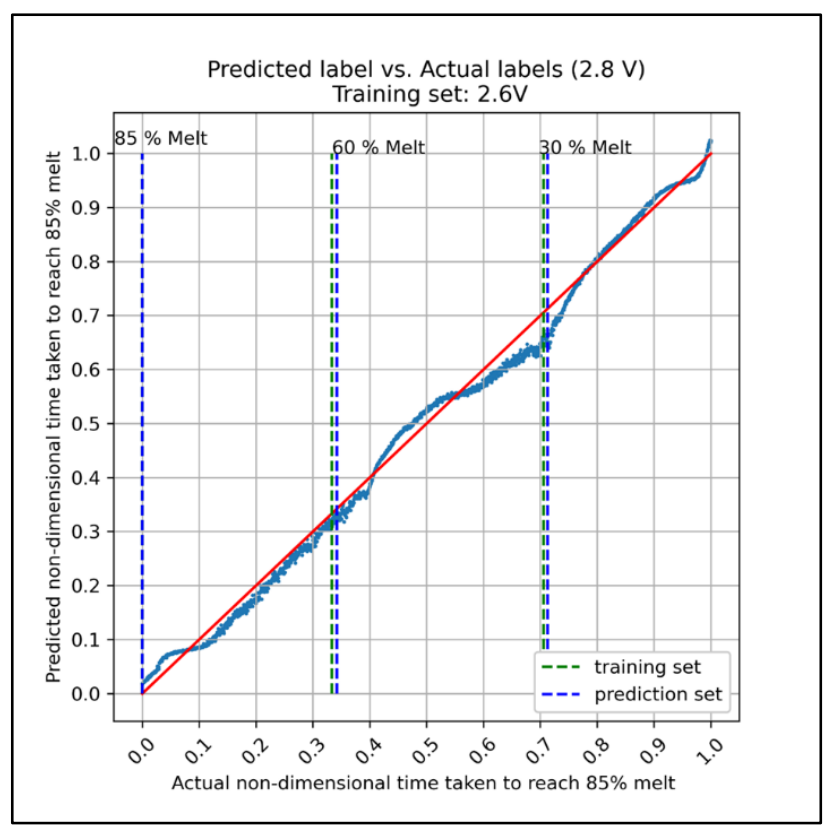

Figure 11. Scatter plot comparing the ANN predictions with experimental data (the actual values for $\tau^{\prime}$ ) for test data set of $2.8 \mathrm{~V}$ (training data set of $2.6 \mathrm{~V}$ ).

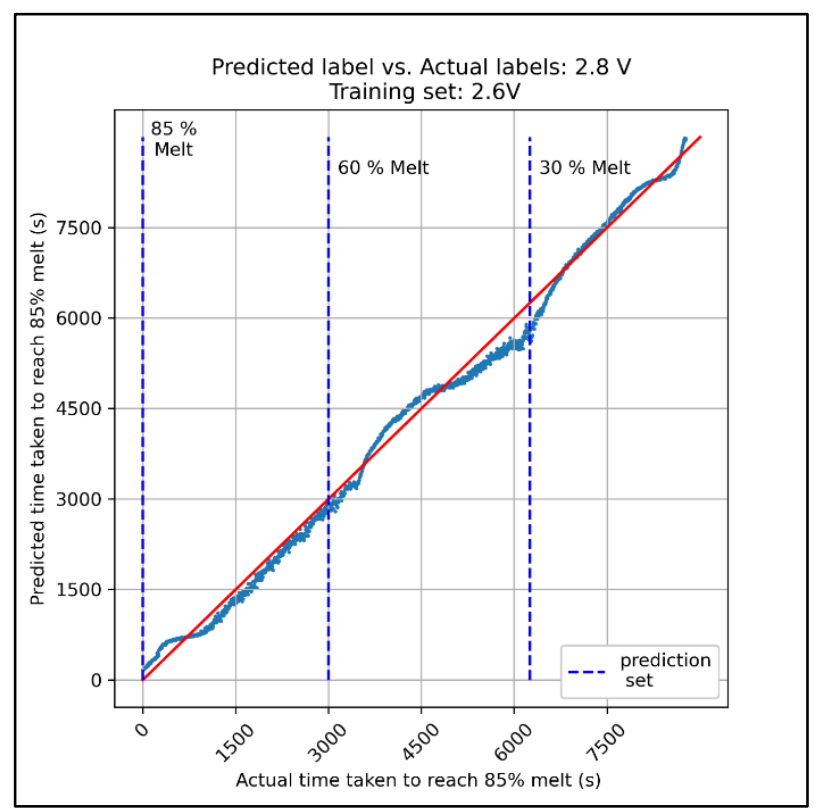

Figure 12. Scatter plot comparing the ANN predictions with experimental data for test data set of $2.8 \mathrm{~V}$ (training data set of $2.6 \mathrm{~V}$ ). 
As another combination, the ANN model is trained on the $2.3 \mathrm{~V}$ set and deployed to predict on the $2.8 \mathrm{~V}$ dataset. The results for the predictions from the ANN model are plotted in Fig. 13. At approximately $3000 \mathrm{~s}$ prior to reaching $85 \%$ melt-fraction, the prediction error is observed to peak to a value of $-1000 \mathrm{~s}$. However, the average absolute error in the last $1800 \mathrm{~s}$ (before reaching $85 \%$ melt fraction) is $331 \mathrm{~s}$, which corresponds to a minute fraction $(\sim 4 \%)$ also. With the training/prediction combination of $2.8 \mathrm{~V} / 2.3 \mathrm{~V}$ the same metric is $653 \mathrm{~s}$ which amounts to $\sim 5 \%$ of the total time to reach $85 \%$ melt-fraction (of the $2.3 \mathrm{~V}$ set). Table 3 summarises the prediction errors for all combinations of training/ prediction data-sets. This ANN method, applied on a simple experimental apparatus, yields prediction errors that are less than 10 minutes, for a forecasting point that is 3 hours ahead of reaching the target melt-fraction of $85 \%$. Hence, the efficacy of the method is established and this demonstrated the feasibility of the ANN model (or MLP model) as well as the computational and experimental techniques that were deployed in this study.

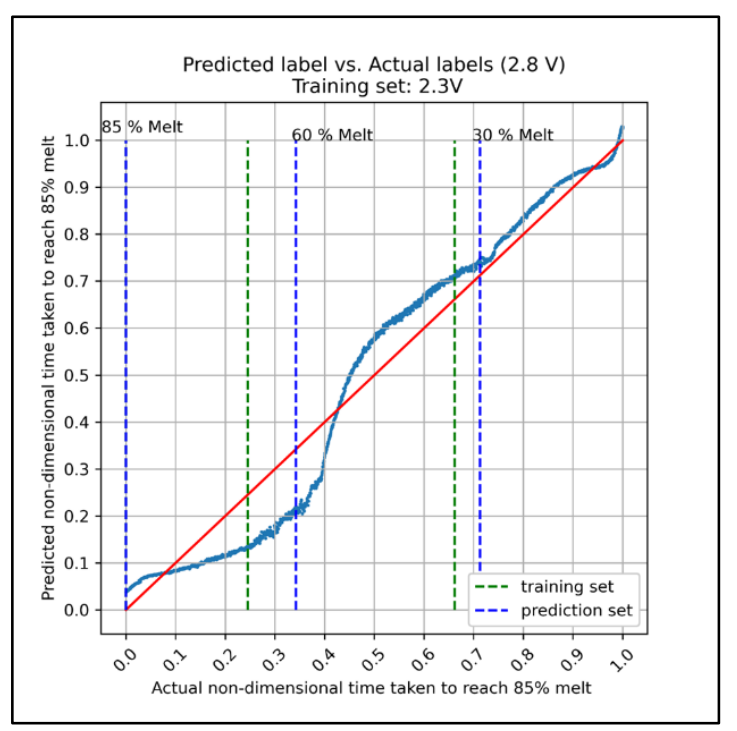

Figure 13. Scatter plot comparing the ANN predictions with experimental data (the actual values for $\tau^{\prime}$ ) for test data set of $2.8 \mathrm{~V}$ (and training data set of $2.3 \mathrm{~V}$ ).

Table 3. Average absolute error in the final $1800 \mathrm{~s}$ (prior to attaining a melt-fraction of $85 \%$ ) for different training/prediction data-set combinations using transient PCM-temperature data.

\begin{tabular}{ccccc}
\hline & \multicolumn{5}{c}{ Prediction Set } \\
\hline \multirow{2}{*}{$\begin{array}{c}\text { Training } \\
\text { Set }\end{array}$} & \multicolumn{2}{c}{$\mathbf{2 . 3 \mathrm { V }}$} & $\mathbf{2 . 6 \mathrm { V }}$ & $\mathbf{2 . 8 \mathrm { V }}$ \\
\cline { 2 - 5 } & $\mathbf{2 . 3 \mathrm { V }}$ & & $190 \mathrm{~s}$ & $331 \mathrm{~s}$ \\
\cline { 2 - 5 } & $\mathbf{2 . 6 \mathrm { V }}$ & $423 \mathrm{~s}$ & & $167 \mathrm{~s}$ \\
\cline { 2 - 5 } & $\mathbf{2 . 8 V}$ & $653 \mathrm{~s}$ & $233 \mathrm{~s}$ & \\
\hline
\end{tabular}

\subsection{Performance of the ANN Trained on Surface Temperatures}

The profile of transient surface-temperature data recorded in these experiments were similar to that of the temperature transients recorded within the mass of PCM (as shown in Fig. 5). Another ANN model is trained using the transient surface-temperature data recorded in the same experiments: and are denoted as $T^{\prime} 30, T^{\prime} 60$, and $T^{\prime} 90$ (as the inputs) instead of the PCM temperatures. The topology of the neural network and the $\tau^{\prime}$ labels are the same. Scatter plots and errors are obtained as outlined in the previous section. Fig. 14 shows the scatter plot for $2.6 \mathrm{~V}$ and $2.8 \mathrm{~V}$ datasets for training and prediction respectively. The surface thermocouples are exposed to higher levels of noise from the ambient environment (as compared to that of the thermocouples immersed in the mass of PCM). As a result, the profile of the transient surface-temperature data is not as smooth (as compared to that of the transient PCM-temperature data). Hence, the fluctuations in 
the predicted values are observed to be higher for the ANN models trained using the transient surface-temperature data. Despite this impediment, the average error in the last $1800 \mathrm{~s}$ is $502 \mathrm{~s}(\sim 6 \%$ of the total cycle time).

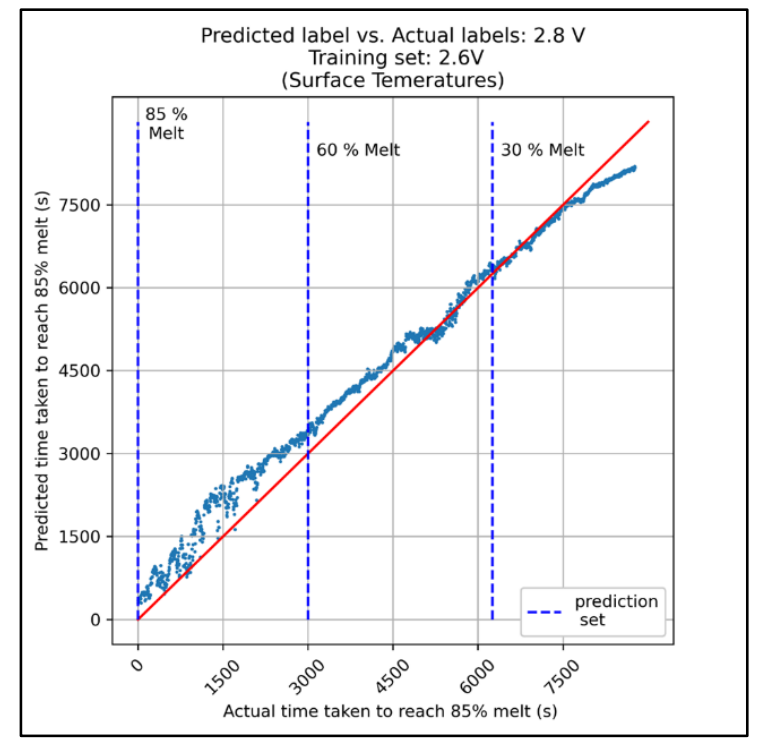

Figure 14. Scatter plot comparing the predictions of the ANN trained on surface temperatures with experimental data for test data set of $2.8 \mathrm{~V}$ (training data set of $2.6 \mathrm{~V}$ ).

The ANN model that was trained using the transient surface-temperature data obtained from the experiments performed for $2.3 \mathrm{~V}$ input was then deployed to obtain predictions for the experiments using $2.8 \mathrm{~V}$ input. The results are plotted in Fig 15. In this case, the deviation is minimized as compared to that of Fig. 13. A summary of the average absolute errors is listed in Table 4. Despite being higher (compared to the magnitude of the errors listed in Table 3), the magnitude of the errors listed in Table 4 are still less than 600 $\mathrm{s}$ (10 minutes), when the real-time forecasting is performed $1800 \mathrm{~s}$ (30 minutes) prior to reaching the target melt-fraction of $85 \%$. Comparison of the tabulated errors (between Table 3 and Table 4), shows that the transient surface-temperature data do not conclusively provide a better strategy for training the ANN models (instead of leveraging the transient PCM-temperature data), specifically for improving the accuracy of the predictions during the final stages of melt-cycle and close to the target values of the melt-fraction. However, the errors are still appreciably low. From a functional standpoint, we can feed surface temperatures to an ANN to predict the time to reach a predefined melt-fraction. It may be noted that underprediction of the time is desirable (it is more reliable) - since over-prediction of the time can cause complete melting of the PCM in the TES - which is a catastrophic failure (since the PCM would then need to be supercooled to achieve nucleation and freezing). For electronics with intermittent operating cycles (i.e., fluctuating duty cycles), this method can be used to leverage the benefits accrued for deploying salt hydrates as PCMs in TES platforms (higher reliability and energy storage capacities with augmented power ratings) while also obviating supercooling. 


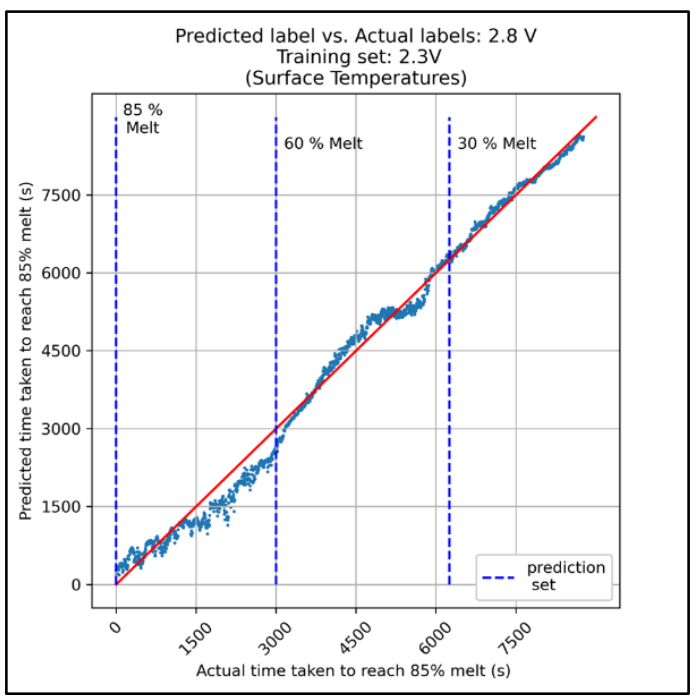

Figure 15. Scatter plot comparing the predictions of the ANN trained on surface temperatures with experimental data for test data set of $2.8 \mathrm{~V}$ (training data set of $2.6 \mathrm{~V}$ ).

Table 4 Average absolute error in the final $1800 \mathrm{~s}$ (prior to attaining a melt-fraction of $85 \%$ ) for different training/prediction data-set combinations using transient surface-temperature data

\begin{tabular}{lllll}
\hline & \multicolumn{4}{c}{ Prediction Set } \\
\hline & \multicolumn{5}{c}{$\mathbf{2 . 3 V}$} & $\mathbf{2 . 6 V}$ & $\mathbf{2 . 8 V}$ \\
\hline \multirow{3}{*}{ Training Set } & $\mathbf{2 . 3 V}$ & & $201 \mathrm{~s}$ & $206 \mathrm{~s}$ \\
\cline { 2 - 5 } & $\mathbf{2 . 6 V}$ & $589 \mathrm{~s}$ & & $502 \mathrm{~s}$ \\
\cline { 2 - 5 } & $\mathbf{2 . 8 V}$ & $279 \mathrm{~s}$ & $447 \mathrm{~s}$ \\
\hline
\end{tabular}

At lower melt-fractions, the prediction errors are lower for ANN model trained using transient surface-temperature data than that of the ANN model trained using PCM temperature data (for target melt fraction of $85 \%$ ). Line graphs are utilised to compare the error in prediction for the two input categories - transient surface-temperature data and transient PCM-temperature data. For instance, as shown in Fig. 16 (training set: $2.3 \mathrm{~V}$, prediction set: $2.6 \mathrm{~V}$ ), for abscissa values in the range of $6,000 \mathrm{~s}$ to $10,000 \mathrm{~s}$; lower values of absolute error is obtained for the predictions from the ANN model trained using transient surface-temperature data, compared to that of the ANN model trained using PCM temperature data. This trend is again evident in Fig. 17 (training set: $2.8 \mathrm{~V}$, prediction set: $2.6 \mathrm{~V}$ ) for abscissa values ranging between 6,500 s and 9,000 s. For yet another case, as shown in Fig. 18 (training set: $2.6 \mathrm{~V}$, prediction set: $2.3 \mathrm{~V}$ ) for abscissa values ranging from $7,000 \mathrm{~s}$ to $12,000 \mathrm{~s}$; the predictions from the ANN model (that was trained using transient surface-temperature data) yield lower magnitudes of the absolute error than that of the ANN model trained using the PCM temperature data. Similar trends are evident in the plots shown in Figs. 19-21. 


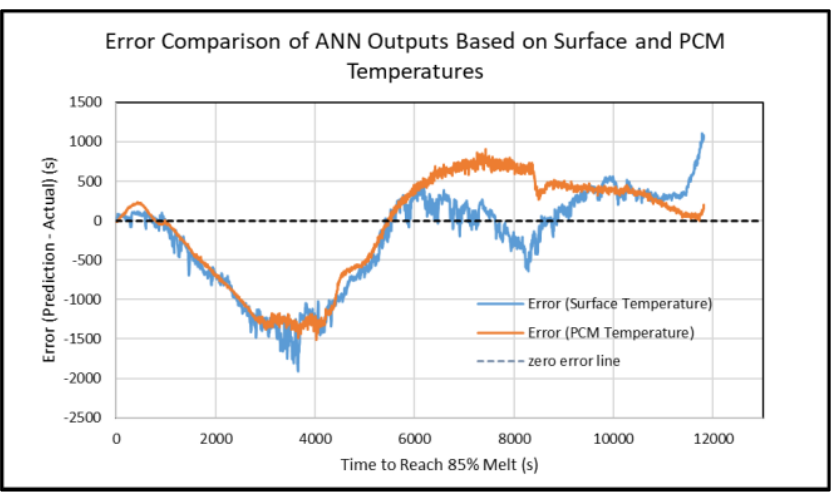

Figure 16. Comparison of error in predictions from the ANN model trained using transient surface-temperature data and transient PCM-temperature data (for test data set of $2.6 \mathrm{~V}$ and training data set of $2.3 \mathrm{~V})$.

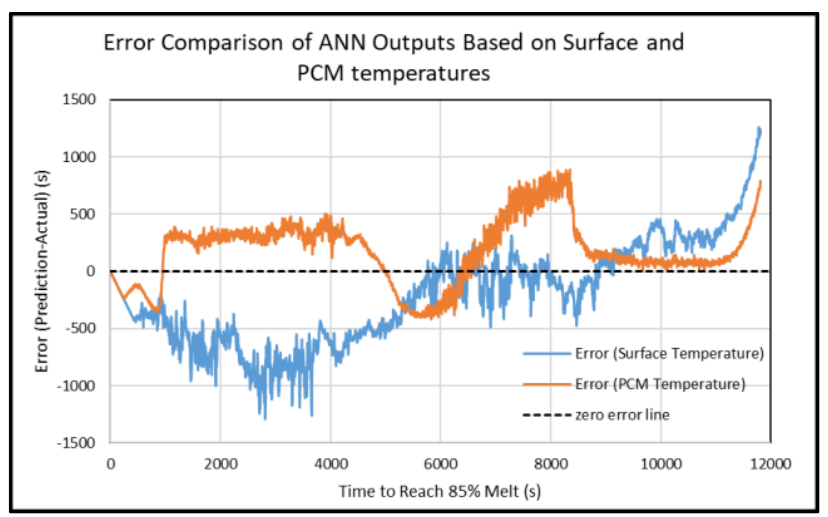

Figure 17 Comparison of error in predictions from the ANN model trained using transient surface-temperature data and transient PCM-temperature data (for test data set of $2.6 \mathrm{~V}$ and training data set of $2.8 \mathrm{~V})$.

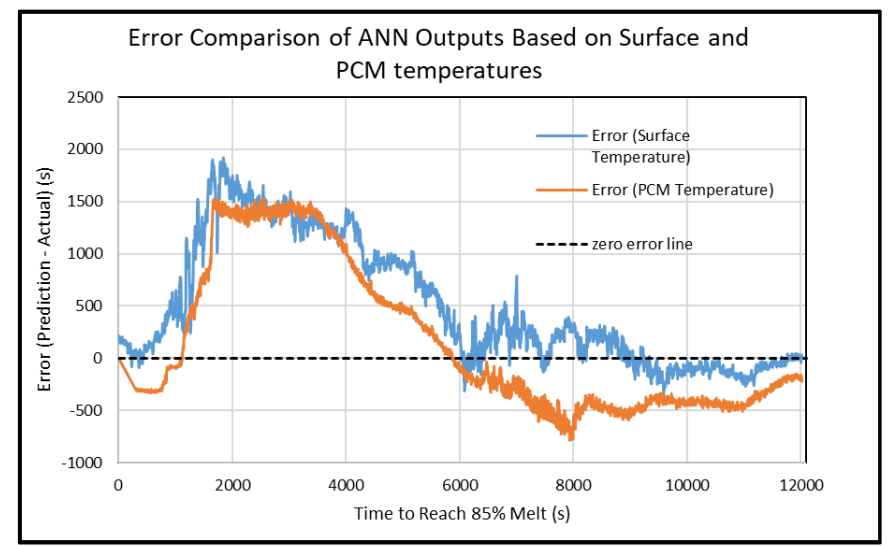

Figure 18 Comparison of error in predictions from the ANN model trained using transient surface-temperature data and transient PCM-temperature data (for test data set of $2.3 \mathrm{~V}$ and training data set of $2.6 \mathrm{~V})$ 


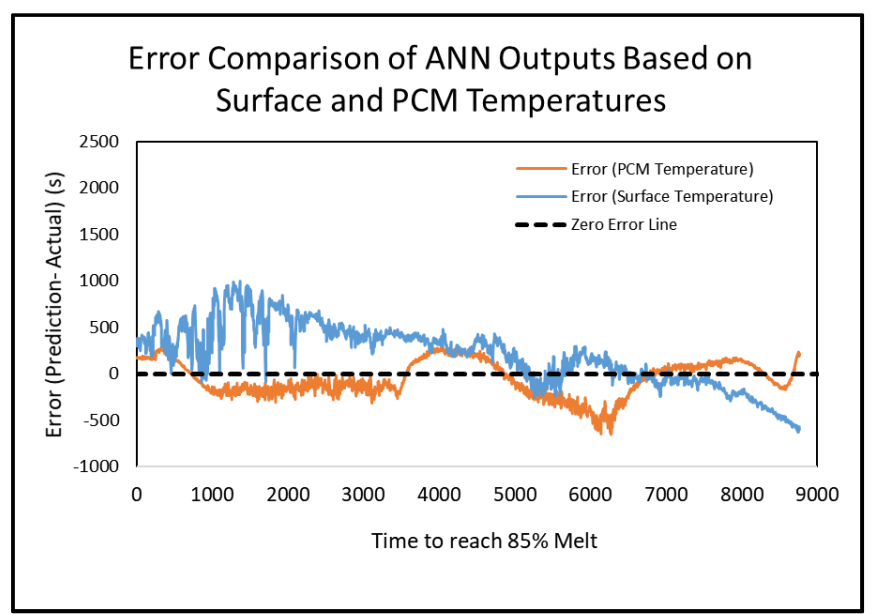

Figure 19. Comparison of error in predictions from the ANN model trained using transient surface-temperature data and transient PCM-temperature data (for test data set of $2.8 \mathrm{~V}$ and training data set of $2.6 \mathrm{~V})$.

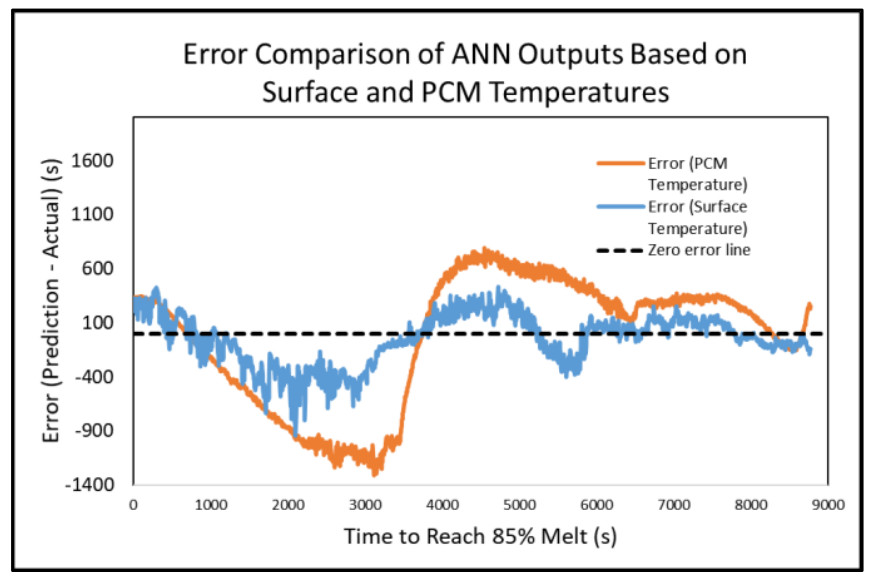

Figure 20 Comparison of error in predictions from the ANN model trained using transient surface-temperature data and transient PCM-temperature data (for test data set of $2.8 \mathrm{~V}$ and training data set of $2.3 \mathrm{~V})$.

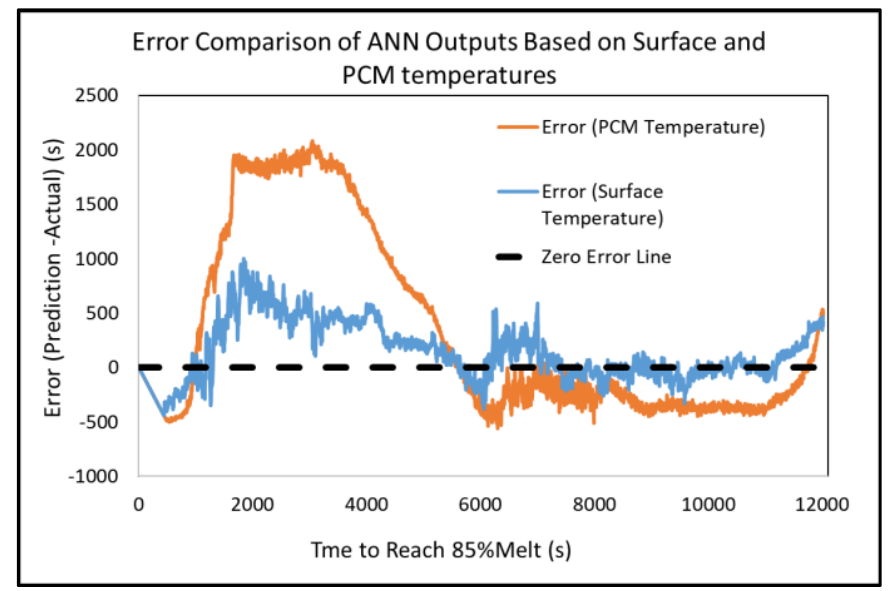

Figure 21. Comparison of error in predictions from the ANN model trained using transient surface-temperature data and transient PCM-temperature data (for test data set of $2.3 \mathrm{~V}$ and training data set of $2.8 \mathrm{~V}$ ). 
Also, it may be noted that for ANN model predictions using transient PCM-temperature data, the error in the predictions are lower when the input power (or input voltage) for the training data is closer to the input voltage of the prediction set. In other words, when $2.6 \mathrm{~V}$ data is used for training, the error in the predictions is lower for $2.8 \mathrm{~V}$ input conditions than that of the $2.3 \mathrm{~V}$ input conditions. Similarly, when the $2.8 \mathrm{~V}$ data is used for training, the error in the predictions is lower for $2.6 \mathrm{~V}$ input conditions than that of the $2.3 \mathrm{~V}$ input conditions.

In contrast, it may be noted that for ANN model predictions using transient surfacetemperature data, the error in the predictions is lower when the input power (or input voltage) for the training data is starkly different compared to the input voltage of the prediction set. In other words, when $2.8 \mathrm{~V}$ data is used for training, the error in the predictions is lower for $2.3 \mathrm{~V}$ input conditions than that of the $2.6 \mathrm{~V}$ input conditions. Similarly, when the $2.3 \mathrm{~V}$ data is used for training, the error in the predictions is lower for $2.8 \mathrm{~V}$ input conditions than that of the $2.6 \mathrm{~V}$ input conditions. This contrast in the prediction capabilities of the ANN model can be attributed to the nature of the training data set itself. On closer observation it may be noticed that the transient surface-temperature data has more acute levels of fluctuations (owing to exposure to free convection to the ambient air) than that of the transient PCM temperature data (owing to the high thermal energy storage capacity locally within the PCM - that tends to damp out any acute temperature fluctuations due to their higher thermal-inertia/ thermal-inductance and thermal-capacitance).

Also, it is worth noting that for ANN model predictions using transient surface-temperature data, the error in the predictions is lower when the input power (or input voltage) for the training data is $2.3 \mathrm{~V}$. In contrast, for ANN model predictions using transient surface-temperature data, the error in the predictions is highest when the input power (or input voltage) for the training data is $2.6 \mathrm{~V}$. Whereas, for ANN model predictions using transient surface-temperature data, the error in the predictions is moderately high when the input power (or input voltage) for the training data is $2.8 \mathrm{~V}$. This anomalous trend can be explained by exploring the nuances of the experimental observations. During the later stages of the melting cycle, especially at higher power input conditions $(2.6 \mathrm{~V}$ and $2.8 \mathrm{~V}$ experiments) - it was observed that the rate of propagation of the melt front was fast enough that it partially lifted the solid mass of PCM above it by a minute amount. This was akin to a hydraulic ram actuation caused by the volumetric expansion due to solidto-liquid phase change as the liquid PCM has lower density than that of the solid phase (and the solid mass of PCM above the melted liquid-phase) acts like a hermetically sealed piston preventing the liquid PCM for leaking up. At which point the plastic tube embedded in the solid mass of PCM caused an opposing reaction force resulting in restoration of the solid mass of PCM back towards the original position. This caused a ripple effect in the temperature transients that were recorded by both the thermocouples embedded in the PCM and by the thermocouples mounted on the surface of the measuring cylinder (this occurred typically towards the end of the melt cycle and at high values of melt fraction, when the melt fraction exceeded $\sim 80 \%$ ). As a result, the training data set for these cases cause distortions in the parameters defining each neuron in the ANN model (i.e., the weight function, the activation function and the bias). Consequently, the predictions from these ANN models display high magnitudes of error and are also less reliable (i.e., often cause over-predictions).

In contrast, at lower power input conditions ( $2.3 \mathrm{~V}$ experiments) - the melt front propagated very slowly (since the power input was barely in excess of the heat loss from the measuring cylinder by free convection). As a result, the solid mass of PCM was virtually undisturbed in these experiments. As a result, there is less distortion in the parameters defining individual neurons. Consequently, the predictions from the ANN model trained using transient temperature data from the $2.3 \mathrm{~V}$ experiments (either for the surface temperature data set or the PCM temperature data set) have better fidelity. Consequently, the magnitude of the error is lower and the predictions are more reliable (i.e., the results tend to underpredict the time required to reach the target value of $85 \%$ melt fraction) for major proportion of the melt cycles. 


\section{Conclusion}

This study proves the feasibility for obtaining real-time predictions using MLP/ ANN models for improving the reliability and efficacy of TES platforms that leverage PCMs. This approach therefore enables the successful deployment of 'Cold Finger Technique' (CFT) to obviate supercooling. Thus, the storage capacity of the TES is maximized while also enhancing the reliability and resilience of the cooling strategy. This is achieved by deploying the MLP/ ANN models for real-time prediction of the time remaining to attain a target (predefined) melt-fraction during the melting-cycle. The target value of melt-fraction chosen in this study is $85 \%$. Experiments were performed using digital data acquisition apparatus (including flow visualization by a digital-camera, I.R. thermography measurements for verification of temperature uniformity in different segments of the experimental apparatus and measurement of temperature transients using thermocouples that were mounted strategically at specific locations corresponding to the location of the liquid meniscus of the PCM for chosen values of melt-fractions). The experiments were performed for power input values of $1.08 \mathrm{~W}, 1.4 \mathrm{~W}$ and 1.6 W. Data sets obtained for a chosen power input condition was used for predicting (and validating) the values for another set of power input conditions. Two different approaches were explored for training the ANN models: (1) by using transient PCM temperature data; and (2) by using transient surfacetemperature data. Lower magnitudes of error in the predicted values were obtained for ANN models trained using transient surface-temperature data corresponding to lower power input conditions (since anomalous temperature transients were minimized at lower power input conditions due to the gradual progression of the PCM upwards into the mass of solid PCM). The predictions were also found to be reliable for major proportion of the melt cycle (i.e., the values were underpredicted).

On the other hand, anomalous decrease in temperature trends were observed for the temperature transients recorded at higher power input conditions (both for surface temperature and PCM temperature data). This was due to the volumetric expansion of the trapped liquid phase (upon melting from the solid phase) which caused the solid mass of PCM above to be displaced up (followed subsequently by a downward motion of the solid PCM caused by the restoring force of the plastic tube adhering to the solid mass of PCM at the top) - resulting in a sharp decrease in the temperature values recorded by thermocouples that were already submerged in the liquid PCM (and for the surface thermocouples that were mounted at similar heights from the base of the measuring cylinder). Consequently, the parameters for each neuron (e.g., weight function, bias, etc.) in the ANN model were likely to be distorted - thus causing higher magnitude of errors in the predicted values and the predictions were unreliable (i.e., due to over-predictions) for major proportion of the melt cycle.

In contrast, for ANN models trained using transient PCM-temperature data, lower magnitudes of error in the predicted values were obtained when the input power of the training data set was similar to that of the predicted data set. For example, lower magnitudes of error were obtained when the training data set from the $2.6 \mathrm{~V}$ experiments were utilized for predicting the temporal values for the $2.8 \mathrm{~V}$ experiments. Similarly, lower magnitudes in error were obtained when the training data set from the $2.8 \mathrm{~V}$ experiments were utilized for predicting the temporal values for the $2.6 \mathrm{~V}$ experiments. In contrast, the magnitudes of error were higher (and the values were over-predicted, i.e., the predictions were unreliable for major proportion of the melt cycle) for the $2.3 \mathrm{~V}$ experiments when either the data set from $2.6 \mathrm{~V}$ or from $2.8 \mathrm{~V}$ were utilized for training the ANN model. In general, the accuracy of predictions afforded by ANN models that were trained using transient surface-temperature data was higher in the initial stages of the cycle (however, higher levels of error are observed for these cases in the final stages of the melting cycle). Future directions of this study include implementing these experimental studies and ANN model development on actual (e.g., "commercially off-the shelf" or "COTS") 
electronics chips that leverage PCM filled heat sinks. Optimization of the neural network parameters also need to be explored.

Motivated by the potential application of inorganic PCMs in TES (that can be deployed for enhancing the reliability and resilience of thermal management devices), especially in electronics chip cooling platforms, in this study the efficacy of deploying deep learning is explored for enhancing the energy storage capacity for CFT (without compromising the power rating) by forecasting the time required to reach a target melt-fraction. In these applications, the direct measurement of PCM temperature is often not possible due to packaging related issues. Measurement of surface-temperature transients are preferable for electronics packages (especially for those that are filled with PCMs) due to their simplicity, low cost, better reliability, as well as ease of manufacturing and fabrication. This strategy can yield lower failure rates while also providing ease of access for maintenance and repair operations. Forecasting strategies based on transient surface-temperature data also enable retrofitting of existing thermal-management platforms in electronics devices thus rendering additional capabilities for real-time predictions using ANN models (e.g., the time remaining for reaching a target melt-fraction of PCMs that are filled inside the electronics package). These capabilities do not typically accrue from strategies involving measurement of temperature transients from sensors that are immersed within the volume of PCM, i.e., from temperature sensors mounted inside the electronics package (instead of the surface-mounted temperature sensors). Hence, this study demonstrates the efficacy of ANN models for electronics chip cooling applications and can be used in conjunction with PCM filled heat sinks to improve their reliability for thermal management applications (e.g., in data centres) while also impacting the sustainability of these data-centres by reducing the usage of water (e.g., reducing the demand for chilled water and, in-turn, the evaporative losses from the cooling towers) as well as the net power consumption by the data centres. Secondary benefits that accrue from such endeavors - include augmented cooling capabilities and enhanced performance of the thermal management platforms, better durability and increased longevity of the computing platforms; since - more effective deployment of the thermal management platforms that leverage such real-time predictions capabilities from the ANN models result in better temperature uniformity (i.e., mitigating the hot-spots in the packages of the electronics chips and within the chips themselves).

Acknowledgments: This work was performed at the Multi-Phase Flows and Heat Transfer Laboratory (MPFHTL) under the supervision of Dr. Debjyoti Banerjee at Texas A\&M University (TAMU), College Station. The authors also acknowledge support from the Texas A\&M Triads for Transformation $\left(T^{3}\right)$ grant funded by the Office of the Vice President for Research (VPR).

Conflicts of Interest: The authors declare no conflict of interest. 

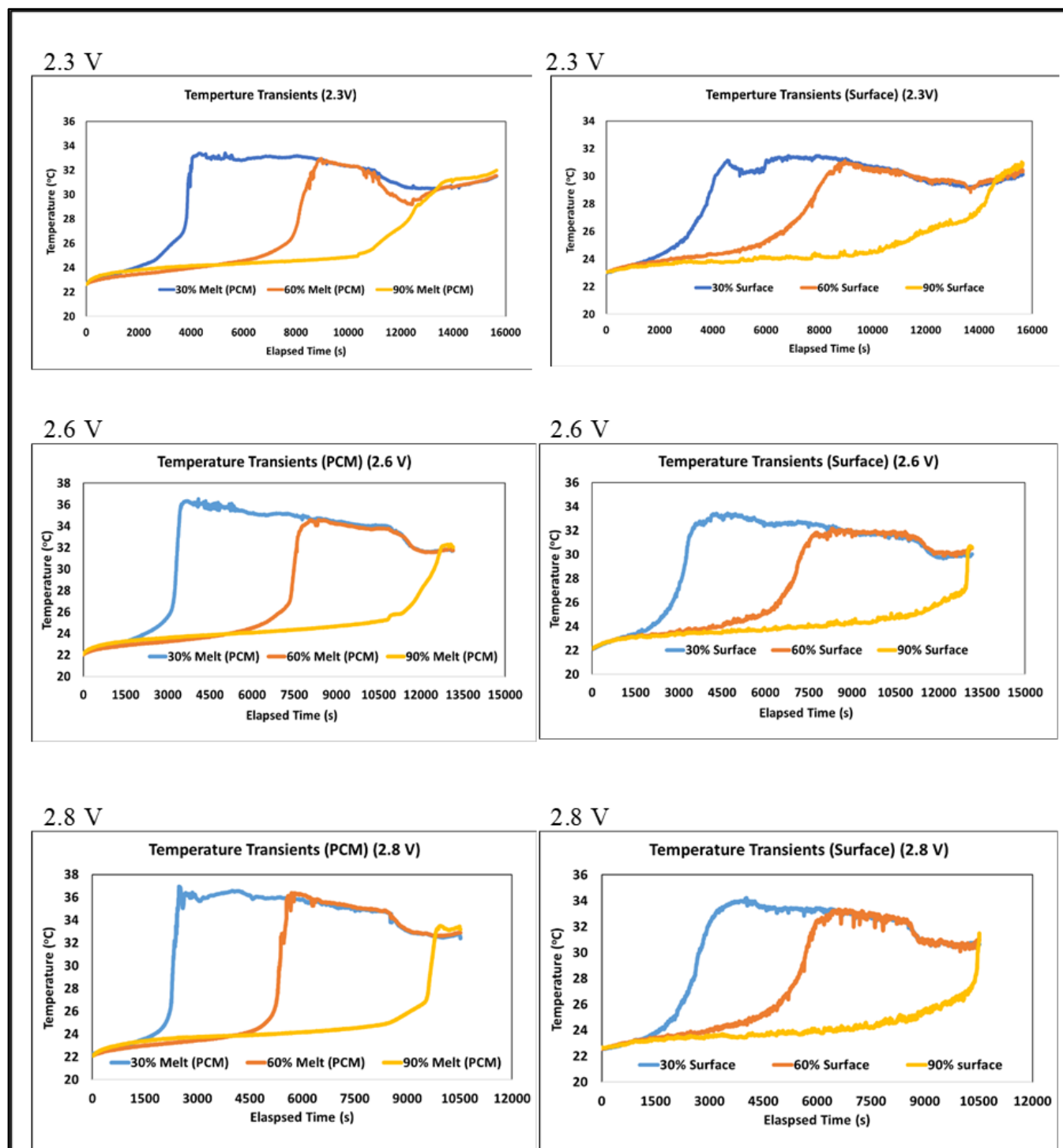

Figure A1. Plots of transient PCM-temperature data and transient surface-temperature data for different heater input voltage (and power input) conditions recorded in the experiments. 


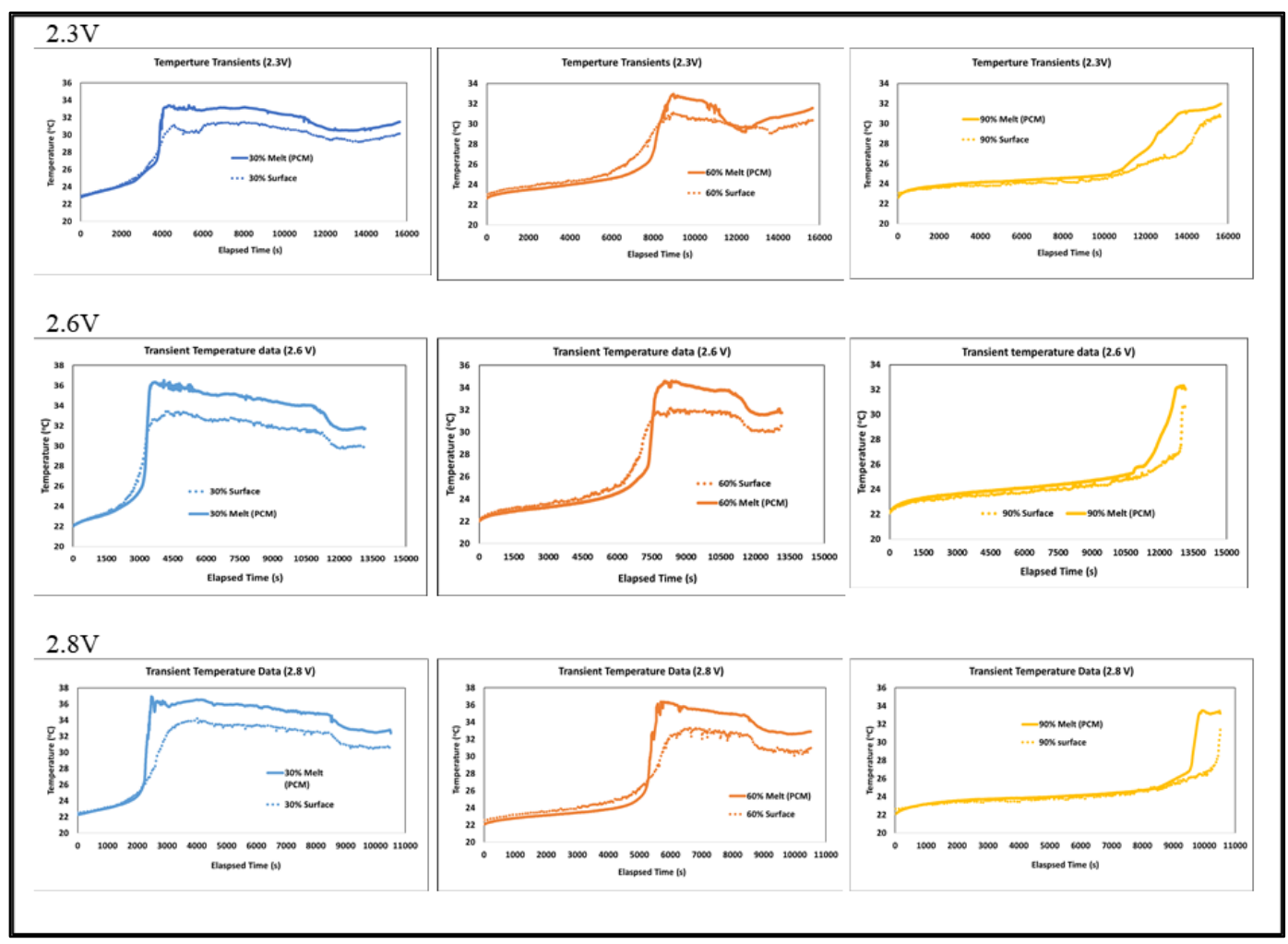

Figure A2. Comparison of the transient PCM-temperature data and transient surface-temperature data for different heater input voltage (and power input) conditions recorded in the experiments.

\section{References}

1. M. Pedram and S. Nazarian, "Thermal Modeling, Analysis, and Management in VLSI Circuits: Principles and Methods," Proceedings of the IEEE, vol. 94, no. 8, pp. 1487-1501, 2006, doi: 10.1109/JPROC.2006.879797

2. J. R. Black, "Electromigration-A brief survey and some recent results," IEEE Transactions on Electron Devices, vol. 16, no. 4, pp. 338-347, 1969, doi: 10.1109/T-ED.1969.16754.

3. G. E. Moore, "Cramming More Components Onto Integrated Circuits," Proceedings of the IEEE, vol. 86, no. 1, pp. 82-85, 1998, doi: 10.1109/JPROC.1998.658762.

4. M. Waldrop, "The chips are down for Moore's law," Nature News, vol. 530, p. 144, 02/01 2016, doi: 10.1038/530144a

5. P. M. Markowski, M. Gierczak, and A. Dziedzic, "Temperature Difference Sensor to Monitor the Temperature Difference in Processor Active Heat Sink Based on Thermopile," Electronics, vol. 10, no. 12, p. 1410, 2021. [Online]. Available: https://www.mdpi.com/2079-9292/10/12/1410.

6. T. Kang, Y. Ye, Y. Jia, Y. Kong, and B. Jiao, "Enhanced Thermal Management of GaN Power Amplifier Electronics with MicroPin Fin Heat Sinks," Electronics, vol. 9, no. 11, p. 1778, 2020. [Online]. Available: https://www.mdpi.com/2079-9292/9/11/1778.

7. S. M. S. Murshed and C. Nieto de Castro, "A critical review of traditional and emerging techniques and fluids for electronics cooling," Renewable and Sustainable Energy Reviews, vol. 78, pp. 821-833, 10/01 2017, doi: 10.1016/j.rser.2017.04.112.

8. Y. Maydanik, S. Vershinin, M. Korukov, and J. Ochterbeck, "Miniature loop heat pipes-a promising means for cooling electronics," IEEE Transactions on Components and Packaging Technologies, vol. 28, pp. 290-296, 2005.

9. X. Wei and Y. Joshi, "Stacked Microchannel Heat Sinks for Liquid Cooling of Microelectronic Components," Journal of Electronic Packaging, vol. 126, no. 1, pp. 60-66, 2004, doi: 10.1115/1.1647124

10. S. V. Garimella, V. Singhal, and L. Dong, "On-Chip Thermal Management With Microchannel Heat Sinks and Integrated Micropumps," Proceedings of the IEEE, vol. 94, no. 8, pp. 1534-1548, 2006, doi: 10.1109/JPROC.2006.879801.

11. M. Jaworski and R. Domański, "A novel design of heat sink with PCM for electronics cooling," in 10th International Conference on Thermal Energy Storage, Stockton, 2006, vol. 31

12. F. L. Tan and C. P. Tso, "Cooling of mobile electronic devices using phase change materials," Applied Thermal Engineering, vol. 24, no. 2, pp. 159-169, 2004/02/01/ 2004, doi: https://doi.org/10.1016/j.applthermaleng.2003.09.005

13. R. Kandasamy, X.-Q. Wang, and A. S. Mujumdar, "Application of phase change materials in thermal management of electronics," Applied Thermal Engineering, vol. 27, no. 17, pp. 2822-2832, 2007/12/01/ 2007, doi: https://doi.org/10.1016/j.applthermaleng.2006.12.013

14. R. Kandasamy, X.-Q. Wang, and A. S. Mujumdar, "Transient cooling of electronics using phase change material (PCM)-based heat sinks," Applied Thermal Engineering, vol. 28, no. 8, pp. 1047-1057, 2008/06/01/ 2008, doi: https://doi.org/10.1016/j.applthermaleng.2007.06.010 
15. R. Baby and C. Balaji, "Thermal management of electronics using phase change material based pin fin heat sinks," Journal of Physics: Conference Series, vol. 395, p. 012134, 2012/11/26 2012, doi: 10.1088/1742-6596/395/1/012134

16. Z. Ling et al., "Review on thermal management systems using phase change materials for electronic components, Li-ion batteries and photovoltaic modules," Renewable and Sustainable Energy Reviews, vol. 31, pp. 427-438, 2014/03/01/ 2014, doi: https://doi.org/10.1016/j.rser.2013.12.017

17. J. Hirschey, K. R. Gluesenkamp, A. Mallow, and S. Graham, "Review of Inorganic Salt Hydrates with Phase Change Temperature in Range of 5 to $60^{\circ} \mathrm{C}$ and Material Cost Comparison with Common Waxes," 2018

18. S. Byung Chul, K. Sang Done, and P. Won-Hoon, "Phase separation and supercooling of a latent heat-storage material," Energy, vol. 14, no. 12, pp. 921-930, 1989/12/01/ 1989, doi: https://doi.org/10.1016/0360-5442(89)90047-9

19. P. Hu, D.-J. Lu, X.-Y. Fan, X. Zhou, and Z.-S. Chen, "Phase change performance of sodium acetate trihydrate with AlN nanoparticles and CMC," Solar Energy Materials and Solar Cells, vol. 95, no. 9, pp. 2645-2649, 2011/09/01/ 2011, doi: https://doi.org/10.1016/j.solmat.2011.05.025

20. B. M. L. Garay Ramirez, C. Glorieux, E. San Martin Martinez, and J. J. A. Flores Cuautle, "Tuning of thermal properties of sodium acetate trihydrate by blending with polymer and silver nanoparticles," Applied Thermal Engineering, vol. 62, no. 2, pp. 838-844, 2014/01/25/ 2014, doi: https://doi.org/10.1016/j.applthermaleng.2013.09.049

21. P. J. Shamberger and M. J. O'Malley, "Heterogeneous nucleation of thermal storage material LiNO3.3H2O from stable latticematched nucleation catalysts," Acta Materialia, vol. 84, pp. 265-274, 2015/02/01/ 2015, doi: https://doi.org/10.1016/j.actamat.2014.10.051

22. N. Kumar, D. Banerjee, and R. Chavez, "Exploring additives for improving the reliability of zinc nitrate hexahydrate as a phase change material (PCM)," Journal of Energy Storage, vol. 20, pp. 153-162, 2018/12/01/ 2018, doi: https://doi.org/10.1016/j.est.2018.09.005

23. N. Kumar, J. Hirschey, T. J. LaClair, K. R. Gluesenkamp, and S. Graham, "Review of stability and thermal conductivity enhancements for salt hydrates," Journal of Energy Storage, vol. 24, p. 100794, 2019/08/01/ 2019, doi: https://doi.org/10.1016/j.est.2019.100794

24. N. Kumar and D. Banerjee, "A Comprehensive Review of Salt Hydrates as Phase Change Materials (PCMs)," International Journal of Transport Phenomena, Article vol. 15, no. 1, pp. 65-89, 2018. [Online]. Available: https://proxy.library.tamu.edu/login?url=https://search.ebscohost.com/login.aspx?direct=true\&db=asn\&AN=131055909\&site=eds-live

25. N. Kumar, R. V. Ness, R. Chavez, Jr., D. Banerjee, A. Muley, and M. Stoia, "Experimental Analysis of Salt Hydrate Latent Heat Thermal Energy Storage System With Porous Aluminum Fabric and Salt Hydrate as Phase Change Material With Enhanced Stability and Supercooling," Journal of Energy Resources Technology, vol. 143, no. 4, 2020, doi: 10.1115/1.4048122

26. S. S. Haykin, "Neural networks and learning machines/Simon Haykin," ed: New York: Prentice Hall, 2009.

27. S. Haykin, "Neural networks: a comprehensive foundation. 1999," Mc Millan, New Jersey, pp. 1-24, 2010

28. A. Chuttar, A. Thyagarajan, and D. Banerjee, "Leveraging Machine Learning (Artificial Neural Networks) for Enhancing Performance and Reliability of Thermal Energy Storage Platforms Utilizing Phase Change Materials." ASME. J. Energy Resour. Technol. February 2022; 144(2): 022001. https://doi.org/10.1115/1.4051048

29. S. Gharbi, S. Harmand, and S. B. Jabrallah, "Experimental comparison between different configurations of PCM based heat sinks for cooling electronic components," Applied Thermal Engineering, vol. 87, pp. 454-462, 2015/08/05/ 2015, doi: https://doi.org/10.1016/j.applthermaleng.2015.05.024

30. PureTemp, “PureTemp29 Technical Data Sheet," https://www.puretemp.com/ stories/puretemp-29-tds 